Cet article a été accepté dans le numéro 32/1 (1er numéro de 2017) de RAM

\title{
La similarité en marketing : périmètre, mesure et champs d'application.
}

\author{
Christophe Bèzes \\ Professeur-chercheur Istec* \\ c.bezes@istec.fr \\ * Istec, 12 rue Alexandre Parodi 75010 Paris \\ Maria Mercanti-Guérin \\ Maître de conférences, CNAM* \\ Dicen \\ maria.mercanti@gmail.com \\ *Cnam, 2 rue Conté, 75003 Paris
}




\title{
La similarité en marketing : périmètre, mesure et champs d'application.
}

\begin{abstract}
Résumé
Le concept de similarité est central dans la recherche en marketing. Ses domaines d'applications sont très variés. Pourtant, ce concept plus complexe qu'il n'y parait, reste encore méconnu. Il doit être distingué d'autres concepts très proches comme la similitude, la différence, le fit et la congruence. Cet article montre que le type de similarité utilisé (littérale ou relationnelle) a des conséquences différentes sur les processus cognitifs des consommateurs. Ceci peut modifier radicalement les résultats d'une expérimentation. Il en va de même du type de mesure utilisée.
\end{abstract} Mots clés : similarité littérale, similarité relationnelle, congruence, fit, extension de marque.

\section{Marketing similarity: scope, measurement and fields of application.}

\begin{abstract}
The concept of similarity is central to marketing research. Its fields of application are very varied. Yet this concept, which is more complex than it seems, is still poorly understood. It must be distinguished from other closely related concepts such as similitude, difference, fit and congruence. This paper shows that the type of similarity used (literal or relational) has differing consequences on consumers' cognitive processes. This can dramatically alter the results of an experiment. The same applies to the type of measurement used.
\end{abstract}

Keywords: literal similarity, relational similarity, congruence, fit, brand extension. 


\section{La similarité en marketing : périmètre, mesure et champs d'application.}

\section{INTRODUCTION}

La similarité est très utilisée en marketing. Elle permet de catégoriser des produits, d'étudier le positionnement concurrentiel d'une marque ou d'évaluer sa capacité à s'étendre vers une autre catégorie de produits. L'importance opérationnelle de ce concept est renforcée par le fait que de nombreuses techniques statistiques y recourent: analyses en composantes principales, regroupements hiérarchiques, méthode des nuées dynamiques,... La similarité permet en effet de catégoriser et de partitionner des groupes d'objets, de valeurs ou de données dans des espaces ou des systèmes en calculant leur proximité, donc leur degré de similarité.

Toutefois, l'opérationnalisation de la similarité en marketing fluctue fortement d'une recherche à l'autre, au moins pour trois raisons. D'abord, la similarité est fréquemment confondue avec les concepts de congruence et de fit (Maille et Fleck, 2011) ; ces trois termes peuvent d'ailleurs coexister dans une même recherche sans être réellement définis. Ensuite, le type de similarité mis en évidence dépend de la manière dont les stimuli ont été préalablement catégorisés par le chercheur. Enfin, des écarts peuvent apparaître selon que la similarité est mesurée « objectivement », c'est-à-dire déterminée par un ensemble de traits ou attributs communs indépendants de l'observateur, ou subjectivement, c'est-à-dire fondée sur des classements « sans critères » visant surtout à établir des catégories (Thibaut, 1997).

Une interprétation aussi fluctuante et contingente de la similarité obère alors la possibilité de comparer de manière fiable les résultats d'une étude à l'autre. Cet article a donc pour objectifs de mieux délimiter le concept de similarité par rapport aux concepts connexes (similitude, différence, fit et congruence), d'en présenter différentes applications pratiques et de montrer de quelle manière des conditions d'une expérimentation peuvent faire varier le type de similarité et sa mesure.

\section{LA SIMILARITE, UN CONCEPT CENTRAL MAIS DUAL}

\section{Un concept central dans les théories de l'apprentissage}

Concept fondamental, omniprésent dans les théories de l'apprentissage et du comportement (Tversky, 1977), la similarité constitue une heuristique qui permet aux individus, « en l'absence de connaissance spécifique, d'établir des suppositions avisées qui les guideront pour 
l'action » (Medin, Goldstone et Gentner, 1993: 258). Dans un environnement complexe, elle rend la pensée humaine plus efficace et plus rapide. En effet, les concepts et les catégories, c'est-à-dire les ensembles (éléments ?) qui constituent la similarité sont au cœur de la cognition humaine (Monneret, 2014). La loi de la similarité régit, par exemple, les rapports de sympathie et d'attraction entre des individus qui présentent des traits de personnalité similaires (Byrne, Griffitt et Stefaniak, 1967). Elle est à ce titre, prédictive des comportements : « si un organisme rencontre dans une nouvelle situation, des éléments semblables à ceux pour lesquels il a déjà donné une réponse, il aura tendance à répéter ses réactions » (Reed, 1927: 111). C'est pourquoi la similarité est fréquemment mobilisée pour étudier les comportements des consommateurs (Ratneshwar et Shocker, 1991 ; Hem et Iversen, 2002), notamment en matière de catégorisation des produits (Cohen et Basu, 1987) et d'extensions de marques.

Sa faculté à (laisser à, plus français que pour) guider les comportements humains s'explique par son rôle clé dans le processus de catégorisation qui permet de réduire la complexité de l'environnement (Ratneshwar et al., 2001). Des choses semblables tendant à être regroupées (Medin, Goldstone et Gentner, 1993), la similarité permet en effet de cerner une catégorie sans même avoir à la définir : "la catégorisation est donc plus une question d'évaluation de la similarité que d'application d'une définition » (Medin et Smith, 1984: 117). C'est d'ailleurs pourquoi la catégorisation holistique, soutenue par des prototypes, définis comme des « images abstraites incorporant les caractéristiques ou attributs les plus usuellement associés aux membres de la catégorie » (Sujan, 1985: 32), ou par des exemplaires (membre de la catégorie qui la définit le mieux), est désormais privilégiée par rapport à la catégorisation analytique à partir d'un ensemble d'attributs (Sujan, Bettman et Sujan, 1986; Fiske et al., 1987). La typicalité qui se définit comme une relation de proximité entre un objet et une classe, ne serait d'ailleurs qu'une «forme déguisée de similarité » (Medin et Smith, 1984: 117) qui assurerait la médiation entre la similarité et la catégorie (Rips et Collins, 1993).

\section{Un concept dual : de la similarité littérale à la similarité relationnelle}

Longtemps, la psychologie et le marketing n'ont considéré la similarité que comme un «type de comparaison » (Medin, Goldstone et Gentner, 1993: 259) qui permet de juger d'une « relation de proximité entre deux objets » (Tversky, 1977: 347). Mais aujourd'hui, la littérature distingue deux grands types de similarité : la similarité littérale (ou taxonomique) et la similarité relationnelle (ou thématique). La distinction entre la similarité basée sur les attributs et celle basée sur les relations est ici fondamentale (Gentner et Gunn, 2001). En effet, «des représentations mentales consistent en des systèmes hiérarchiques qui codent des objets, des 
attributs d'objets », mais aussi «des relations entre objets et des relations entre relations » (Gentner et Markman, 1994: 152). La similarité littérale est dominée par les caractéristiques des objets alors que la similarité non-littérale est plus large et plus profonde (Gentner et Gunn, 2001). Par exemple, une bière Budweiser est littéralement similaire à un cola Budweiser, mais relationnellement similaire à des chips Budweiser car elles sont souvent consommées ensemble (Estes et al., 2012). Seul le terme « similarité littérale » sera utilisé dans la suite de l'article.

\section{La conception initiale de la similarité : la similarité littérale}

La similarité littérale correspond à la conception initiale de Tversky (1977). Formalisée dans le modèle des contrastes, elle marque un net progrès par rapport au modèle de distance mentale de Shepard (1962) ou de Rips, Shoben et Smith (1973). Fondée sur les propriétés tangibles des objets comparés, et notamment sur leurs attributs les plus saillants, elle repose sur l'intuition que plus des objets partagent des caractéristiques communes, plus ils sont similaires (Tversky, 1977). Les attributs y sont définis comme des composantes primitives et insécables des stimuli (Medin, Goldstone et Gentner, 1990). Indépendantes de l'objet, ces propriétés peuvent être concrètes ou abstraites (Gregan-Paxton et John, 1997). En effet, Tversky (1977: 329) ne nie pas que ces caractéristiques «puissent refléter des attributs abstraits tels que la qualité ou la complexité »; les caractéristiques physiques des produits demeurent néanmoins prépondérantes. Allant au-delà de la simple apparence qui ne compare que des attributs de surface, la similarité littérale compare à la fois les attributs et les relations étroites que ces attributs génèrent. Elle épouse la conception de Leibniz (1995) et des mathématiciens qui se référent à des attributs géométriques et concrets, directement proportionnels et dotés de composantes essentielles identiques.

Outil privilégié de la catégorisation, cette similarité littérale sert de base à la théorie probabiliste (Rosch et al., 1976) et à celle de l'exemplaire. Toutefois, si la catégorisation par similarité littérale de Tversky s'est substituée à la théorie classique, plus lourde en matière de verbalisation, de connexions causales et d'explications (Murphy et Medin, 1985), elle a été rapidement remise en cause par d'autres faisant appel à des niveaux d'abstraction plus élevés.

\section{La conception élargie de la similarité : la similarité relationnelle ou thématique}

Plusieurs courants de recherche se sont émancipés du modèle des contrastes, jugé trop restrictif car trop centré sur des caractéristiques perceptuelles (plutôt que conceptuelles). En effet, « la similarité implique beaucoup plus qu'un simple calcul effectué sur un ensemble de caractéristiques fixes... (Elle) aligne des caractéristiques plutôt que de juste les additionner » 
(Medin, Goldstone et Gentner, 1993: 275). Le poids des attributs et des relations a un impact direct sur le type de similarité utilisé (Goldstone, Medin et Gentner, 1991).

Les premiers développements d'une conception élargie de la similarité remontent à Ortony (1979). Il les justifie par l'incapacité de la similarité littérale à relier des domaines jusqu'ici jugés trop éloignés et trop différents. En s'appuyant non plus sur des attributs identiques, mais sur des attributs «hautement similaires » (Ortony, 1979: 167) ou sur des relations entre attributs, voire entre objets, Ortony (1979) multiplie les degrés de similarité depuis la forme la plus littérale où tous les attributs saillants et identiques coïncident indépendamment du contexte (« les panneaux d'affichage ressemblent aux affiches »), à des formes non littérales comme les métaphores (« les panneaux d'affichage ressemblent à des verrues ») qui peuvent se révéler « non pertinentes ou inappropriées » (Ortony,1979: 162) dans un contexte particulier. Même si elles n'y font pas explicitement référence, trois conceptions non-littérales de la similarité découlent des propositions d'Ortony.

La théorie de la cohérence conceptuelle comble certaines lacunes du modèle des contrastes en termes de catégorisation. En effet, la similarité littérale est trop sensible aux pondérations des attributs, au contexte et aux instructions données lors de l'expérimentation (Medin, Goldstone et Gentner, 1993). C'est pourquoi elle n'est pas « suffisamment contraignante pour déterminer la cohérence d'un concept » et est donc incapable d'expliquer à elle seule, la formation d'une catégorie (Murphy et Medin, 1985: 289). En s'inspirant du raisonnement par analogies, la théorie de la cohérence conceptuelle permet de maximiser le chevauchement et les différences conceptuelles entre les objets comparés. Elle suggère que le jugement de similarité consiste à rechercher le meilleur alignement structurel possible des informations activées : le concept ou la catégorie doit présenter dans sa structure interne des «propriétés connectées par des relations fonctionnelles ou par un schéma causal », et ne doit pas contredire la base de connaissances déjà acquises sous peine d'être « instable ou rapidement oubliée » (Murphy et Medin, 1985: 313). Les individus peuvent d'autant mieux juger de la similarité que les différences sont alignables, c'est-à-dire reliées à une structure commune (Gentner et Markman, 1997 ; Goldstone, Medin et Gentner, 1991) et plus mémorisables (Loken, 2006). Le recours à l'alignement structurel des différences aboutit à un jugement plus fin et plus sûr que le modèle des contrastes (Medin, Goldstone et Gentner, 1993). D'ailleurs, pour Murphy et Medin (1985: 313), « la forte similarité entre membres de la catégorie et la faible similarité entre catégories n'est qu'un sous-produit de la structure interne ». Dans le cas où les objets comparés présentent 
peu de caractéristiques communes et ne peuvent donc pas être alignés, alors l'intégration relationnelle s'impose (Bassok et Medin, 1997). Deux théories y font référence.

Barsalou valide la structure hiérarchisée des catégories ad hoc en utilisant une conception relationnelle, donc moins stricte de la similarité. En se basant sur les usages (Ratneshwar et Schocker, 1991 ; Ratneshwar et al., 2001) ou sur les buts (Barsalou, 1983 et 1985), cette théorie part du principe que les individus peuvent constituer spontanément des catégories pour atteindre un but dans un contexte nouveau. Ces catégories, qui ne sont révélées que par un contexte particulier, diffèrent des catégories naturelles parce qu'elles ne sont pas logées dans la mémoire à long terme (sauf si elles sont fréquemment mobilisées) et parce qu'elles peuvent «inclure des produits disparates qui partagent peu ou pas de caractéristiques de surface » (Ratneshwar et al., 2001: 148). Par exemple : des chips et des glaces comme prototype de produits anti-diététiques (Boush, 1997).

Une dernière approche se concentre sur une forme extrême de relations : les relations thématiques. La différence essentielle entre les catégories ad hoc et les relations thématiques tient au fait que les premières sont organisées autour d'un but commun que les propriétés internes communes à chaque objet (Estes et al., 2012) permettent de servir de manière pertinente (Barsalou, 1983). Sans ce but commun, aucune cohérence entre ces objets n'existe. En revanche, les relations thématiques sont totalement extérieures aux objets et reposent sur des éléments qui doivent jouer des rôles très différents et complémentaires dans un même thème ou scénario. Dans ce cas, la complémentarité semble presque fortuite : par exemple, interaction inattendue mais réussie d'un iPod et d'une chaussure dans les Nike+ (Estes et al., 2012).

Cette conception duale (littérale mais aussi relationnelle) de la similarité brouille les frontières avec certains concepts voisins, en premier lieu avec celui de congruence.

\section{LA SIMILARITE FACE AUX CONCEPTS PROCHES}

Le sens commun considère les jugements de similarité comme de parfaits symétriques des jugements de différence. Il n'en est rien (Goodman, 1972). En 1913, Hollingworth constatait déjà ce phénomène : « le plus semblable n'est pas ... le moins différent, pas plus que le moins similaire est identique au plus différent » (Hollingworth, 1913: 289). En effet, « la similarité est une relation où se combinent différence et identité » (Monneret, 2014: 7). Tversky (1977), Medin, Goldstone et Gentner (1990) ou Dubé et Schmitt (1999) montrent ainsi que les jugements de similarité confèrent plus d'importance aux caractéristiques communes alors que les jugements de différence se focalisent sur les points distinctifs. Les premiers sont aussi plus 
globaux et moins analytiques que les seconds, ce qui les rend plus faciles, plus naturels et plus cohérents dans le temps (Hollingworth, 1913). La distinction entre ces deux concepts pose en premier lieu le problème des échelles de mesure utilisées pour évaluer tout autant la similarité que la différence (Medin, Goldstone et Gentner, 1990). Sur un plan plus managérial, elle explique aussi pourquoi, selon qu'ils sont évalués en termes de similarité ou bien de différence, des produits proches qui partagent des attributs communs (Coca et Pepsi plutôt que jus de fruits ou bière) seront jugés tout à la fois comme plus similaires et plus dissimilaires que les autres (Johnson, 1981).

A l'inverse, la similarité est souvent confondue à tort avec les concepts de similitude, de congruence et de fit.

\section{Similarité et similitude}

Bien que possédant la même étymologie, la similarité n'est pas synonyme de similitude. En effet, la similitude désigne une ressemblance complète (identité) entre deux entités alors que la similarité renvoie à une ressemblance approchante (Thibaut, 1997). En se fondant sur plusieurs similitudes, la similarité est donc plus globale et moins exacte que la similitude. Par ailleurs, contrairement à celle-ci, la similarité implique toujours un sujet (l'objet considéré) et un référent (l'objet auquel il est comparé). Ce dernier est généralement favorisé pour sa saillance (Tversky, 1977 ; Dubé et Schmitt, 1999), c'est-à-dire pour son intensité et sa prévalence dans la classification utilisée à ce moment-là (principe de «diagnosticité »). Le recours obligatoire à un référent explique d'ailleurs pourquoi, contrairement au jugement de similitude en mathématique, le jugement de similarité en psychologie est toujours « directionnel » (Tversky, 1977: 328), c'est-à-dire non transitif et asymétrique. La stratégie de positionnement par similarité d'un nouvel entrant peut ainsi échouer lorsque les consommateurs ont du mal à attribuer « un nombre limité de bénéfices clairement identifiables » au leader référent (Dubé et Schmitt, 1999: 212) ; un nouvel entrant aura au contraire davantage de chances de réussite s'il capitalise sur un attribut unique très distinctif (Cunha et Laran, 2008). Parce que la propriété « asymétrique de la similarité implique le transfert asymétrique de l'affect » (Cunha et Laran, 2008 : 425), Boush (1997) préconise, par exemple, qu'une marque nouvelle cultive d'abord sa réputation sur le produit prototypique de la catégorie avant de s'étendre à d'autres produits de la même catégorie ad hoc. Cette propriété de la similarité a aussi été utilisée dans le domaine de la publicité comparative par Johnson et Horne (1987) et dans celui des produits hybrides par Saaksjarvi et Pulkkinen (2009). 
Enfin, pour que l'objet comparé puisse «entrer en correspondance » avec son référent (Kruhmans, 1978: 452), la similarité est toujours construite par l'individu. Cette capacité d'infléchir à sa guise ce jugement permettra toujours à une vieille tante de trouver à un nouveauné quelque air de ressemblance avec ses propres aïeux (Shanon, 1988).

\section{Similarité et fit}

Dans la littérature en marketing, le concept de similarité n'est quasiment jamais délimité par rapport à des concepts connexes tels que ceux de congruence et de fit. Il est même le plus souvent confondu avec eux. Pourtant, il s'agit initialement de concepts bien distincts.

En tant que concept (et non comme liaison entre deux concepts), le fit est presque exclusivement utilisé en marketing pour évaluer l'opportunité d'une extension de marque qui peut se définir comme le fait d'utiliser une marque établie pour lancer un nouveau produit ou service dans une catégorie différente de la marque-mère (plutôt que dans la même catégorie : extension de gamme). Ainsi, il y a fit «quand le consommateur accepte le produit comme étant logique et s'attend à voir ce produit sous cette marque » (Tauber, 1988: 28). Pour cet auteur, il constitue avec l'effet de levier (capacité de la marque à s'imposer dans la catégorie en extension), l'une des deux conditions de réussite du transfert d'attitude vis-à-vis de l'extension. Mais la conception du fit varie fortement au fil des recherches, puisqu'il peut s'agir d'un « lien direct ou indirect en terme de type de produit (même technologie ou savoir-faire de fabrication, produits complémentaires), des attributs physiques et/ou fonctionnels, des cibles ou des bénéfices consommateur en commun, ou encore des évocations symboliques (statut social,...) » (Dimitriadis, 1993: 26).

Les premiers travaux sur l'extension de marque (Boush et al., 1987) ont d'abord introduit la similarité entre les catégories de produits pour modérer le fit, c'est-à-dire le transfert d'affect entre l'évaluation globale de la compagnie et une nouvelle marque que celle-ci a créée. En effet, l'affect généré par une marque ne suffisait pas à lui seul pour garantir une extension atypique. L'extension est alors d'autant moins bien perçue que la similarité entre les catégories de produits est faible et que l'image de la marque est excellente mais très spécialisée.

Quelques travaux, minoritaires en nombre mais pas en intérêt, maintiennent cette distinction conceptuelle entre la similarité et le fit, en faisant du premier concept non plus un modérateur, mais un antécédent du second. Pour Park, Lawson et Milberg (1989), les jugements de similarité se distinguent des jugements de fit car ils opèrent au niveau des produits (attributs concrets et caractéristiques fonctionnelles), et non pas au niveau des marques (valeurs 
symboliques et concepts abstraits). Tout comme Broniarczyk et Alba (1994), ces auteurs identifient deux antécédents du fit : l'un lié à la similarité entre produits ou entre catégories de produits ; l'autre lié à la perception de cohérence du concept de la marque en extension, c'està-dire aux associations induites par la marque. La distinction entre similarité, cohérence du concept et fit expliquerait selon eux, pourquoi les extensions de marques de prestige (plutôt que fonctionnelles) pourraient présenter un bon fit sans que pour autant il y ait similarité d'attributs, de propriétés ou d'usages entre leur catégorie d'origine et celle en extension. Prolongeant le travail de Czellar (2003), Bèzes et Dubois (2013) montrent que la similarité relie des croyances alors que le fit relie des attitudes en y apportant un sens, c'est-à-dire un jugement de valeur. Les croyances étant les antécédents des attitudes, la similarité pourrait donc être un antécédent du fit comme Park, Milberg et Lawson (1991) l'ont modélisé.

Les recherches qui persistent à dissocier les deux concepts relativisent aussi beaucoup plus que les autres le rôle de la similarité en matière d'extension de marque : sauf pour certains produits, la similarité n'agirait pas directement sur l'évaluation de l'extension mais indirectement via le fit (MacInnis et Nakamoto, 1991, in Cegarra et Merunka, 1993: 66). L'impact du fit serait davantage lié aux associations spécifiques de la marque par rapport à ses concurrentes qu'à la similarité (Park, Milberg et Lawson, 1991 ; Broniarczyk et Alba, 1994). En effet, si les jugements de similarité sont susceptibles de faciliter les jugements de fit (Park, Milberg et Lawson, 1991), Broniarczyk et Alba (1994) doutent que les consommateurs aient besoin de procéder à un jugement de similarité globale entre les deux catégories. Pour ces auteurs, peu importe que les produits soient similaires ou pas en termes d'attributs ; ce qui compte c'est qu'ils correspondent aux bénéfices escomptés dans une situation donnée (similarité relationnelle et non pas littérale). D'ailleurs, plus la marque est associée aux attributs de son produit d'origine moins elle semble extensible hors de sa catégorie initiale ; au contraire, plus l'utilité de la marque est reconnue, plus celle-ci est extensible (Rangaswamy, Burke et Oliva, 1993). D'où l'intérêt de repérer le type de similarité à l'œuvre dans ce processus (Hem et Iversen, 2009).

Cependant, un autre courant de recherche, toujours dominant, a très vite assimilé la similarité au fit, mais sans définir précisément ce qu'il entendait par similarité (parmi les auteurs les plus connus : Aaker et Keller 1990, Dacin et Smith 1994 ou Brudvig et Raman 2006). Les travaux d'Aaker et Keller (1990) et de Keller et Aaker (1992) sont emblématiques de cette conception, équivoque car indéfinie, de la similarité. La complémentarité des deux classes de produits (capacité à « être consommées en même temps et pour un même besoin ») et la substituabilité 
(capacité à se remplacer l'un l'autre et « à satisfaire les mêmes besoins ») sont les deux dimensions finalement retenues par ces auteurs, comme constitutives du fit, donc de la similarité. Or, la substituabilité repose sur une similarité concrète donc littérale alors que la complémentarité fait référence à une similarité abstraite et relationnelle car fondée sur des usages ou sur des buts (Taylor et Bearden, 2002 ; Mao et al., 2012). En outre, la conception du fit chez Aaker et Keller est fluctuante puisque le fit est d'abord relationnel (Aaker et Keller, 1990) puis littéral (Keller et Aaker, 1992). D’où la nécessité pour Bridges (1989), Bridges, Keller et Sood (2000) ou Seltène (2004), de le scinder en fit produits/extension fondé sur une similarité littérale, et en fit image/extension, fit association et fit contexte, tous quatre fondés sur une similarité relationnelle ou une congruence.

La nécessité de mieux distinguer la similarité du fit d'une part, et la similarité littérale de la similarité relationnelle d'autre part, rend donc les contributions pionnières de Park, Milberg et Lawson (1989 et 1991), de MacInnis et Nakamoto (1991), de Broniarczyk et Alba (1994) ou de Herr, Farquhar et Fazio (1996) aujourd'hui très pertinentes en matière d'extensions de marques.

\section{Similarité et congruence}

Ce besoin de clarification conceptuelle est tout aussi urgent pour distinguer la similarité de la congruence. Pourtant, la plupart des exemples de congruence étudiés en marketing portent sur des objets qu'il serait impossible de comparer dans le cadre de la similarité de Tversky (1977) : une marque et un produit (Meyers-Levy, Louie et Curren, 1994), un parrain et l'événement auquel il s'associe (Kamins et Gupta, 1984), des éléments verbaux et picturaux dans une publicité (Heckler et Childers, 1992), l'image de l'utilisateur d'un produit et l'image de soi (Sirgy, 1982), le goût et l'odeur d'un produit alimentaire (Maille, 2007), une musique et une publicité (Kellaris et al., 1993 ; Galan, 2009) ou des sites marchands et des magasins (Bèzes, 2013).

Théorisée par Mandler (1982) et opérationnalisée en marketing par Meyers-Levy et Tybout (1989), la congruence est définie « comme la cohérence descriptive entre des attributs et des attentes associées, des contextes, ou des schémas »(Kirmani et Shiv, 1998: 44) et l'incongruence comme « une violation des attentes » (Bruner et Postman, 1949: 208). La notion de schéma mental qui supporte des attentes et des prévisions est donc centrale dans le concept de congruence (Mandler, 1982 ; Meyers-Levy et Tybout, 1989) : c'est par rapport à ce schéma mental que l'on peut juger si la congruence est pertinente et/ou attendue. Les 
schémas sont « des structures organisées de connaissances représentant des concepts tels que des situations, des objets, des événements, et des actions à différents niveaux d'abstraction. Les fonctions centrales des schémas sont d'autoriser la compréhension des données entrantes courantes et de prédire les événements futurs »(Schützwohl, 1998: 1183). Par exemple, Meyers-Levy et Tybout (1989) testent l'incongruence entre un soft drink traditionnel (schéma initial) et un soft drink composé d'ingrédients totalement naturels.

En revanche, la similarité fait davantage référence aux catégories. Or, les schémas diffèrent des catégories parce qu'il s'agit de «structures spatialement et temporellement organisées » (Stayman, Alden et Smith, 1992: 240) qui intègrent comme les catégories, « des connexions verticales entre une catégorie supérieure (nom de la catégorie) et une catégorie subalterne (membre) », mais aussi «des connexions horizontales basées sur la relation temporelle, spatiale et causale » (Khan et Paivio, 1988: 558). C'est d'ailleurs l'activation du schéma associé qui déclenche l'effort de catégorisation (Pavelchak, 1989).

Une deuxième distinction entre la similarité et la congruence concerne les types de stimuli mis en relation. La congruence implique une comparaison sur une base extrinsèque (plutôt qu'intrinsèque) aux objets et moins concrète que la similarité (Leibniz, 1995). En effet, elle met en jeu des équivalences indirectes entre objets pouvant être de natures différentes, alors que la similarité implique une comparaison directe entre attributs ou objets semblables et homogènes, c'est-à-dire de même nature (Bèzes, 2010). Cette capacité de la congruence à rapprocher des entités de natures très différentes s'explique par sa faculté à explorer des relations structurelles plus profondes, toujours par analogie donc avec des niveaux d'abstraction plus élevés et moins d'attributs partagés que la similarité (Leibniz, 1995 ; Ortony, 1979 ; Gentner et Markman, 1997 ; Gregan-Paxton et John, 1997 ; Bèzes, 2010).

A ce titre, la congruence intègre ou combine les stimuli en présence alors que la similarité les compare. Grâce à ce mécanisme d'intégration, le premier objet présenté (le référent) ne biaise pas le jugement de congruence (Cech, 1989), ce qui rend ce jugement symétrique et transitif, au contraire du jugement de similarité. Dans sa dimension «pertinence », la congruence pourrait même dans certains cas, intégrer un jugement antérieur de similarité littérale (Maille et Fleck, 2011).

\section{Synthèse}

La figure 1 montre que la plupart des concepts précédemment discutés se différencient essentiellement par le nombre d'attributs concrets communs et de relations communes qu'ils prennent en compte. La similitude porte sur un attribut commun partagé par les deux objets 
comparés alors que la similarité prend en compte un faisceau d'attributs concrets partagés, donc de similitudes. De son côté, la congruence se focalise sur des relations plus abstraites entre ces objets, ce qui lui permet de relier entre eux des objets moins proches physiquement. En revanche, le fit se distingue de la congruence car il intervient au niveau des attitudes, et non pas des croyances. Bèzes et Dubois (2013) prennent l'image de deux prises de courant, l'une mâle, l'autre femelle: bien que n'ayant aucune caractéristiques physiques communes (absence de similarité littérale), elles partagent des propriétés d'usage (congruence) qui peuvent selon les cas, se traduire par un bon ou un mauvais fit. Sur l'axe « croyances-attitudes », la congruence est positionnée plus haut que la similarité littérale ; elle peut en effet relier des croyances intermédiaires (bénéfices ou buts) et ainsi intervenir plus tard dans le processus de décision du consommateur.

(Insérer Figure 1)

Finalement, l'extension du concept de similarité, au-delà de la seule comparaison des attributs (Tversky, 1977), pourrait l'avoir rendu « vide de sens » (Medin, Goldstone et Gentner, 1993 : 254 se référant à Goodman, 1972). En effet, la similarité relationnelle n'est-elle pas simplement de la congruence ? Etonnamment, cette question n'est quasiment jamais abordée dans la littérature en psychologie ou en marketing. Seul Barsalou (1983), dans son expérimentation 2b, hésite sur le processus d'élaboration des catégories ad hoc: comparaison par similarité ou bien, une autre relation d'équivalence sans comparaison (comme la congruence)?

Au-delà de cette zone d'ombre conceptuelle, un consensus se dégage néanmoins sur trois points qui font de la similarité un processus «dynamique » (Medin, Goldstone et Gentner, 1993: 275) : 1/ le jugement de similarité est davantage construit par l'individu que déterminé par les caractéristiques des objets en présence ; 2 / un modèle dual de similarité, mesuré par les attributs et/ou sur les relations, s'est imposé chez les psychologues. En effet, deux processus distincts peuvent agir dans (Bassok et Medin, 1997) ou sur (Gentner et Gunn, 2001) les jugements de similarité : l'un compare les propriétés des stimuli lorsqu'elles sont d'une manière ou d'une autre alignables; l'autre les intègre relationnellement dans un scénario commun, notamment lorsque ces stimuli sont difficilement alignables ; 3/ la nature des stimuli en présence conditionne le processus cognitif mis en œuvre : comparaison et non pas intégration (Bassok et Medin, 1997).

Les différentes conceptions de la similarité auxquelles peuvent se référer les chercheurs interdisent d'aller au-delà de la figure 1 sous peine de présenter une vision restrictive ou 
contestable du concept; l'extrême diversité de ses champs d'application aussi. Ce concept est en effet transverse à toutes les politiques marketing, depuis la gestion du produit jusqu'à la communication, en passant par la créativité des processus ou des productions (lancement de nouveaux produits ou services). La seconde partie de l'article dresse un panorama synthétique de son utilisation en marketing en distinguant l'influence interpersonnelle de la similarité (action sur la personne), sa capacité à dégager de nouvelles voies créatives tant dans la communication, la marque ou le produit (action sur le processus), son apport dans l'analyse des champs concurrentiels ou culturels nouveaux pour l'entreprise (action sur l'environnement). Par la suite, nous présenterons les principaux modérateurs de la similarité et les principaux outils disponibles pour la mesurer.

\section{LES CHAMPS D’APPLICATION DE LA SIMILARITE EN MARKETING}

La similarité est très utilisée en marketing en tant que mesure ou en tant que concept. L'une de ses applications les plus connues concerne l'analyse des marchés et de la concurrence. Elle est aussi fréquemment mobilisée pour étudier la relation client-vendeur ou les extensions de marques.

\section{La similarité au fondement des analyses des marchés et de la concurrence}

Chandon et Strazzieri (1986) soulignent le lien conceptuel entre ensemble évoqué et similarité. Compte tenu de leur similarité, les marques concurrentes exercent le même attrait et la même configuration de bénéfices pour le consommateur. Pham (1996) utilise ainsi un mapping des similarités à deux dimensions (distance et qualité) pour illustrer quatre effets de contexte essentiels dans le choix d'un restaurant (effet d'attraction, de contraste, de compromis et d'humeur).

La similarité des marchés est aussi considérée comme le préambule à une stratégie d'internationalisation efficace. Il s'agit ici d'identifier des marchés similaires pour adapter le mix-marketing à ces zones mais aussi réaliser des économies d'échelle. Hofstede, Wedel et Steenkamp (2002) segmentent les marchés internationaux en prédéfinissant des zones géographiques supposées homogènes en termes de styles de vie, de valeur et donc de comportements de consommation. En utilisant une approche bayésienne hiérarchique, ils développent un modèle général. De même, Douglas et Craig (1996) analysent les similarités entre unités géographiques et portefeuilles d'activités ; pour eux, les indices d'une bonne similarité inter-marchés résident dans un comportement des consommateurs (goûts, intérêts, achats) et un environnement identiques (régulation, distribution, infrastructures, publicité). Grein (2000) 
propose une mesure de la similarité entre marchés fondée sur l'évaluation du PIB par tête et un score de proximité culturelle adaptée des travaux d'Hofstede. Il montre que la publicité et la détermination des prix sont les variables du mix les plus influencées par la similarité de marchés. Dans une optique plus organisationnelle, Kim et Parkhe (2009) distinguent, quant à eux, la similarité de compétition et la similarité de coopération. Ils proposent une échelle de mesure à quatre dimensions (contexte culturel, contexte national, corporate, pratiques managériales).

Du côté du consommateur, l'étude de la concurrence recourt à trois concepts clés : la substituabilité, la similarité et l'intensité de concurrence reposant sur l'ensemble de considération (Mercanti-Guérin, 2010). Si la substituabilité varie selon la situation d'usage envisagée par le consommateur, la similarité est en revanche stable d'une situation à l'autre et se fonde sur l'asymétrie entre marques. Cette asymétrie est liée au fait que les marques d'un même marché sont considérées comme des sous-ensembles d'attributs de la marque prototypique. Les recherches étudient principalement les relations de la similarité sous l'angle concurrentiel et son influence sur les heuristiques décisionnelles.

Bien que les jugements de préférence ne puissent pas être assimilés à des jugements de similarité, certains auteurs considèrent qu'ils s'influencent très fortement (Derbaix et Sjöberg, 1994). Néanmoins, les jugements de préférence chez les consommateurs reposeraient sur l'analyse des bénéfices utilitaires ou hédoniques alors que les jugements de similarité reposeraient sur l'analyse des attributs physiques d'un produit (Creusen et Schoormans, 1997). Même si ces auteurs n'y font pas allusion, ce résultat pourrait illustrer à quel point la similarité littérale centrée sur les attributs concrets des objets considérés n'agit pas au même niveau du processus d'élaboration et de décision des consommateurs qu'une similarité relationnelle davantage centrée sur des bénéfices ou des buts. La similarité littérale constitue vraisemblablement une croyance de base alors que la similarité relationnelle constitue une croyance intermédiaire plus subjective, ce qui explique la forte part d'affectivité repérée par Collange (2005) dans les jugements de préférence. Parce que, pour cette raison, les marques les plus littéralement similaires ne sont pas obligatoirement les plus concurrentes, Chandon et Strazziéri (1986) recommandent d'ailleurs d'utiliser de façon combinatoire des cartes perceptuelles concurrentielles et des cartes perceptuelles fondées sur la similarité.

Dans le domaine de la communication également, la similarité est considérée comme une variable essentielle de la concurrence entre marques. La recherche de Poncin, Pieters et Ambaye (2006) fondée sur la théorie de l'assimilation-contraste montre que les annonces placées dans 
un même écran publicitaire sont d'autant plus concurrentes qu'elles sont perçues comme similaires. Elle prouve que les réactions positives associées tant à la dimension plaisir qu'à la dimension excitation sont influencées par les réactions affectives déclenchées par l'annonce précédente en cas d'annonces dissimilaires. Par ailleurs, plus l'implication est forte à l'égard du produit, plus les annonces sont traitées de façon systématique et plus la similarité ou la dissimilarité peuvent être perçues comme importantes. Pour sa part, Law (2002) analyse la répétition de slogans de marques perçues comme similaires et leur impact sur la mémoire. Elle met en évidence la confusion mémorielle induite par la répétition de slogans de deux marques similaires et insiste sur l'importance de ses résultats dans un contexte concurrentiel aboutissant aux lancements de produits me-too. La répétition publicitaire dans un contexte de marché encombré a ainsi pour effet une neutralisation des concurrents entre eux.

Schweidel, Bradlow et Williams (2006) étudient la manière de faire évoluer la communication d'une marque dans le temps et en fonction de la concurrence, tout en conservant les attributs spécifiques qui ont fait sa force (sagas publicitaires). Ils proposent un modèle de mesure de la similarité qui prend en compte un score de similarité élaboré en fonction de publicités présentées par paires, une évaluation de la distance entre les items publicitaires et le noyau central (items spécifiques conservés quelle que soit la communication). Seules trois dimensions apparaissent explicatives de la perception de la similarité entre annonces : l'humour, les caractéristiques-produit et l'impression de continuité entre les annonces.

La similarité est aussi utilisée en matière de publicité comparative. Celle-ci est définie comme une exécution publicitaire qui copie les attributs produit spécifiques dans le but d'associer les caractéristiques physiques de la nouvelle marque avec les caractéristiques physiques de la marque dominante (Wilkie et Farris, 1975). La publicité comparative est plus efficace pour détourner vers une nouvelle marque les acheteurs d'une marque dominante qu'une imitation de son packaging ou de ses attributs-produit (Dröge et Darmon, 1987). En recourant au modèle des contrastes, Johnson et Horne (1988) montrent en effet que la publicité comparative renforce les associations entre marques et accroît leur similarité perçue.

Si la littérature donne une place importante à l'utilisation de la similarité par les médias traditionnels, le Web en fait aussi un concept central dans la construction d'algorithmes de recommandation sociale et personnalisée. Celle-ci se fonde sur une mesure multidimensionnelle de la similarité sur la base de critères individuels en termes d'âge ou de genre (Gefen et Straub, 1997) ou en termes de motivations d'achat (Hassanein et Head, 2006). Outre cette similarité littérale, elle prend aussi en compte des similarités plus relationnelles, en comparant les parcours de navigation, les recherches effectuées ou les sites visités. La similarité 
est ainsi utilisée pour recommander l'achat de mots clés : des algorithmes, comme ceux utilisés par Google Adwords, calculent des indices de similarité contextuelle entre les mots clés achetés par les annonceurs et les pages Web sur lesquelles vont s'afficher les publicités. Elle est aussi mobilisée pour insérer sur les sites, des publicités Display : les bannières d'un annonceur sont adressées aux internautes ayant manifesté un intérêt pour des sites similaires en termes de produits ou de contenus. Plus récemment, le look-alike modeling a étendu ce ciblage comportemental aux prospects les plus fortement similaires aux bons clients. Cette modélisation repose sur le principe que «Vos meilleurs prospects ressemblent à vos meilleurs clients ».

\section{La similarité interpersonnelle : un objet d'études aux multiples applications}

Un autre champ d'application de la similarité en marketing concerne la similarité interpersonnelle. Celle-ci constitue une des causes principales de l'attraction ressentie entre deux individus. Cette attraction interpersonnelle comporte plusieurs dimensions dont l'intelligence perçue, la désirabilité ou la proximité physique (Byrne et Griffith, 1973). Mobilisée en marketing dans la conception de produits, la distribution, la communication ou la gestion de la force de vente, la similarité interpersonnelle a fait l'objet de recherches assez disparates tant en termes de mesures que de conclusions. Elle a été étudiée sous l'angle strictement interpersonnel (similarité entre consommateurs) ou sous un angle plus transitif (similarité entre consommateurs via le produit ou similarité entre consommateurs et représentants de la marque). Ici, nous lui conférons un statut plus large de relation : relation entre clients, entre clients et force de vente, mais aussi entre individus et avatars.

Les effets de la similarité entre deux individus sont relativement aisés à démontrer. L'hypothèse de similarité-attraction prédit en effet que des personnes similaires ont plaisir à être ensemble (Nass et al., 1995). Néanmoins, les effets de la similarité interpersonnelle ne sont pas toujours positifs. Swartz (1984) montre ainsi qu'il n'existe pas de relation entre l'expertise perçue de l'émetteur et la similarité perçue avec le récepteur. Cette absence de relation doit donc être nuancée en fonction de la catégorie de produits. Dans le domaine des cosmétiques, la perception d'une faible similarité avec le mannequin présent dans la publicité, aboutit à une faible expertise perçue. Pour des produits plus technologiques et peu liés au corps comme l'achat d'une télévision, il existe un lien entre faible expertise et forte similarité comme si l'expert ne pouvait ressembler au novice. Dernièrement, Bourcier-Bequaert et De Barnier (2014) ont montré que la similarité d'âge subjectif entre un mannequin dans une annonce 
publicitaire et des consommatrices seniors agit indirectement sur l'attitude envers la marque, mais que cette similarité perçue a un effet indirect sur l'attitude envers la marque ; cet effet est renforcé si les consommatrices peuvent s'identifier aisément à un mannequin « ordinaire ». En matière de contexte d'achat, la seule présence de consommateurs similaires à soi influence la satisfaction induite par le shopping, via un processus tant affectif que cognitif (Kwon et Ha, 2013). Zhang et al. (2014) étudient comment les facteurs sociaux peuvent influencer la visite en magasin. Ils mettent en évidence que les contacts du client avec d'autres clients mais aussi des vendeurs modifient le temps et le parcours d'achat du consommateur. Le processus d'achat est davantage influencé par les interactions clients-clients que par les interactions clientsvendeurs. Néanmoins, en cas d'interactions clients-vendeurs, la similarité perçue avec la force de vente (notamment au niveau de l'âge, du genre et de l'ethnie) a une influence sur les comportements d'achats. Jiang et al. (2010) montrent ainsi que la similarité dite incidentale (partager le même prénom, la même date de naissance, vivre dans le même quartier) entre consommateurs et vendeurs a une influence favorable sur les attitudes et les intentions d'achat en magasin. Lorsque l'âge ou l'ethnie est similaire, le client a moins de contact physique avec le produit. Il éprouve un besoin plus faible de réassurance ce qui n'est pas le cas lorsque le genre est identique. Cette influence est modérée par le type d'interactions entre clients et par le nombre de clients présents sur le point de vente.

Concernant la conception de produit, Damak (1997) effectue des mesures morphologiques (réelles et perçues) en rapport avec le design de flacons de parfum. Sa recherche confirme la tendance à préférer des formes physiquement similaires chez les individus qui ont une image positive de leur corps (concept de soi) et des formes différentes ou complémentaires chez ceux qui en ont une image négative (volonté de rapprocher son soi perçu de son soi idéal). Si dans la recherche de Damak (1997), on peut encore parler de similarité, il convient davantage de parler de congruence (Sirgy, 1982) dans les recherches qui traitent de l'image d'un produit et du concept de soi, puisque les objets comparés ne sont pas du tout de même nature.

L'interface individu-site Internet fait également appel à une similarité avec de nouvelles perspectives conceptuelles. La similarité peut ainsi être vue comme une similarité physique, de mouvement ${ }^{1}$ (même façon de se déplacer pour un individu et son avatar) ou sociale (sentiment de même appartenance sociale entre l'avatar et l'individu). Cette similarité étendue

\footnotetext{
${ }^{1}$ La similarité de mouvement est difficile à manipuler et ses effets suivent une courbe en $\mathrm{J}$ inversée. Plus un robot ressemble à un être humain, plus les émotions à son égard sont positives jusqu'au moment où on quitte la similarité pour se rapprocher d'une imitation plus ou moins parfaite de l'homme. Les sentiments négatifs croissent, alors, et se focalisent sur les dernières différences restantes jugées très sévèrement (Mori, 1970).
} 
passe par une vision anthropomorphique de l'objet comparé à l'individu (Nowak, 2004). L'internaute tend à personnifier son ordinateur (Moon, 2000), d'où la tendance à socialiser et à humaniser les contacts sur les sites Internet pour améliorer l'expérience en ligne (Lemoine et Cherif, 2012). C'est ainsi que les rapports qu'entretiennent les consommateurs et les avatars sont étudiés sous l'angle de la similarité interpersonnelle. David-Ignatieff (2012) montre que la similarité perçue d'âge entre le consommateur et l'avatar a une influence sur l'achat ${ }^{2}$. Nass et al. $(1995)^{3}$ prouvent que les internautes privilégient les avatars de même apparence ethnique qu'eux, qu'ils leur font plus confiance et jugent la qualité de leurs arguments plus élevés que des avatars moins similaires.

Générer de la similarité interpersonnelle représente donc un vrai enjeu pour les sites de ventes en ligne mais aussi pour les distributeurs et leurs forces de vente. Ce défi les amène à créer de nouvelles formes d'interaction et à imaginer d'autres formes de communication ou de distribution.

\section{La similarité comme source de créativité en marketing}

La similarité est de plus en plus utilisée par les praticiens pour donner une cohérence à leur politique marketing dans le cadre de lancements de produits, d'adoption de formes de communication inédites mais aussi d'extension de marque ou de canal de distribution. Elle peut même être considérée comme un fondement de la créativité. En effet, un consommateur créatif est un individu qui dispose d'un réseau cognitif dense lui permettant de relier des concepts entre eux (Hirschman, 1980). Le réseau inter-concepts est mesuré en soumettant au consommateur une liste diversifiée de produits représentatifs de différents domaines de consommation (transports, loisirs, nourriture). Ces produits sont arrangés par paire, et les individus doivent lister les similarités et les ressemblances à l'intérieur de chaque paire. Plus le consommateur sait déceler des liens de similarité ou de «dissimilarité » entre les produits ou les différents attributs de ces produits, plus sa créativité est jugée forte, car son aptitude à résoudre un éventuel problème de consommation est accrue par sa capacité à effectuer une comparaison la plus exhaustive possible des différents produits et à sélectionner un produit supérieur. Un consommateur expérimenté dispose donc d'un réseau inter-concepts plus développé qu'un consommateur moins expérimenté. La créativité liée au produit trouve une

\footnotetext{
${ }^{2}$ La similarité perçue avec un avatar est également désignée par le terme d'homophilie (Mc Croskey et Richmond, 1979 ; Lemoine et Cherif, 2012). Lazarsfeld (1954) définit l'homophilie comme le degré de similarité perçue entre l'observateur et l'agent observé. David-Ignatieff (2012) s'appuie sur deux types d'homophilie : l'homophilie de statut (âge, sexe, religion, emploi) ou l'homophilie de valeurs et d'attitudes.

${ }^{3}$ Cités par David-Ignatieff (2012).
} 
partie de ses fondements dans le modèle associationniste de Mednick et Mednick (1962). Décrite comme une façon d'atteindre une solution créative, la similarité créative est considérée par ces auteurs comme le fondement d'arts tels que la rhétorique ou la musique.

Gill et Dubé (2007) étudient la création de nouveaux concepts de produits à partir de la combinaison de deux concepts de produits déjà existants. Les concepts de nouveaux produits créés en combinant relationnellement des concepts existants sont plus rapidement et plus facilement interprétés que ceux créés en associant littéralement les concepts. Parmi les concepts créés de manière littérale, ceux qui combinent ou modifient les fonctions de deux types de produits (plutôt que leurs caractéristiques non-fonctionnelles) sont aussi plus difficiles à interpréter. L'hybridation courante aujourd'hui de produits technologiques pose donc des problèmes cognitifs aux consommateurs ; les entreprises doivent les prévenir en investissant davantage dans l'explication et dans la publicité.

Une autre application de la similarité dans le domaine de la créativité concerne la co-création de produits. Par exemple, Thompson et Malaviya (2013) examinent les conditions dans lesquelles une annonce publicitaire dont la création a mobilisé des consommateurs est efficace auprès de consommateurs non impliqués dans sa conception. En général, une telle initiative est source de scepticisme eu égard aux compétences des co-créateurs. Thompson et Malaviya (2013) montrent que mettre en avant des consommateurs co-créateurs a seulement un effet positif sur les consommateurs aux ressources cognitives limitées, très fidèles à la marque et qui partagent certains traits communs à ceux des co-créateurs.

Mais concernant la création de nouveaux produits, la similarité est surtout utilisée dans les extensions de gammes et de marques. Ce thème a déjà été largement abordé pour distinguer conceptuellement la similarité du fit; nous y ferons à nouveau référence lorsque seront examinées les variables qui conditionnent les mécanismes de la similarité et ses effets.

\section{LES MESURES ET FACTEURS DE CONTINGENCE DETERMINANTS DE LA SIMILARITE}

\section{Les mesures de la similarité}

Les méthodes pour mesurer la similarité sont presque aussi variées que ses applications. Il peut s'agir de méthodes qualitatives ou quantitatives indirectes qui, à partir de traits ou de dimensions communes, permettent d'analyser les proximités entre objets comparés. Il peut aussi s'agir de méthodes directes, plus subjectives, qui recourent à des échelles pour mesurer une similarité globale ou des similarités plus spécifiques (littérale ou bien relationnelle). Si les mesures indirectes paraissent plus objectives, elles ont néanmoins l'inconvénient de prendre 
en compte des antécédents de la similarité que l'individu n'aurait pas forcément considérés de prime abord (Maille et Fleck, 2011).

Le tableau 1 dresse un panorama non exhaustif de ces instruments de mesure par domaines d'application. Il exclut les outils qui ne font pas explicitement référence au concept de similarité, mais plutôt à ceux de congruence ou de fit. C'est pourquoi par exemple, les travaux d'Aaker et Keller ne sont pas ici répertoriés ici alors qu'ils le sont dans la synthèse livrée par Estes et al. (2012) et à laquelle le lecteur peut se reporter.

(Insérer Tableau 1)

Seules les recherches sur les extensions de marque n'utilisent que des échelles de mesure ; Les études qui portent sur d'autres secteurs utilisent tout aussi bien des mesures indirectes que directes. Cette diversité de mesure de la similarité indépendamment du domaine étudié constitue indéniablement une opportunité d'enrichissement et d'approfondissement pour les chercheurs qui sauront les croiser ou les associer. En outre, les techniques de référencement naturel $^{4}$ sur le Web font désormais appel à des indices de similarité. Leur objectif est de distinguer le contenu original du contenu copié ou plagié, peu valorisé par les moteurs de recherche tels que Google. Ces méthodes, qui analysent des ensembles de mots, présentent des points communs avec les analyses sémantiques utilisées en marketing et traitant de la similarité : elles analysent des ensembles de mots $^{5}$ et mettent en avant les similarités entre deux pages Web.

Cette extrême diversité d'outils peut néanmoins être source de complexité en faisant apparaître des écarts inexpliqués de résultats selon la méthode utilisée. Mais au-delà de divergences de mesures, ces écarts peuvent être dus à des facteurs personnels ou contextuels.

\section{Les facteurs de contingence de la similarité}

Plusieurs facteurs sont susceptibles de faire varier la perception de la similarité mais aussi ses conséquences sur le comportement des consommateurs :

\footnotetext{
${ }^{4}$ Le référencement naturel regroupe l'ensemble des techniques qui améliore la position d'un site dans les résultats des recherches des moteurs comme Google ou Bing.

${ }^{5}$ L'indice généralement utilisé est celui de Jaccard, qui permet d'étudier la similarité entre des objets constitués d'attributs binaires et particulièrement les ensembles d'objets. Cet indice consiste à diviser le nombre d'objets communs aux deux ensembles par le nombre d'objets distincts. Webrankinfo et Seoquake, qui sont des outils de référencement naturel, proposent ainsi des calculs de similarité.
} 
- la manière dont les stimuli sont présentés. Par exemple, plus les stimuli seront présentés sous forme d'images plutôt que décrits verbalement, plus leurs caractéristiques distinctives prennent d'importance (Medin, Goldstone et Gentner, 1990) ;

- le type de comparaison demandée et l'option focale qui impose une direction, c'est-àdire le choix d'un sujet et d'un référent (Tversky et Gati, 1982) ;

- la situation (Barsalou, 1983 ; Corfman, 1991 ; Schmitt et Dubé, 1992) ;

- les caractéristiques personnelles des individus (Sujan, 1985 ; Alba et Hutchinson, 1987).

Les types de jugements de similarité mobilisés pour évaluer des extensions de marque permettent d'illustrer de manière concrète ces différents points.

Tout d'abord, les catégories d'objets à comparer lors d'une expérimentation peuvent involontairement biaiser les résultats en orientant les répondants vers tel ou tel type de similarité. Les consommateurs recourent à la similarité relationnelle lorsqu'ils doivent mettre en relation des catégories d'objets éloignées en termes de contenu et que la marque mère de ces produits est prestigieuse (Monga et John, 2010). En effet, cette mise en relation nécessite d'examiner des éléments plus abstraits (Johnson, 1984 ; Gentner, 1988 ; Ozanne et al., 1992 ; Markman et Wisniewski, 1997). C'est ce qui explique que selon Park, Lawson et Milberg (1989), pour des produits fonctionnels, l'extension de marque fonctionne mieux si le nouveau nom de marque est associé à des éléments concrets et fonctionnels. Le consommateur part des attributs produits pour juger de la pertinence de l'extension (bottom-up). A contrario, pour des produits symboliques, le consommateur réagira plus favorablement aux extensions lorsque le nouveau nom de marque véhicule des éléments de prestige. Dans ce cas, son jugement part de l'abstrait, c'est-à-dire de la marque (top-down) pour évaluer la pertinence de l'extension. C'est ce qui explique que selon Park, Lawson et Milberg (1989), pour des produits fonctionnels, le nom de marque (le nom de la marque mère étudiée) est le résultat final du jugement à partir des éléments concrets (bottom-up), alors que pour des produits symboliques, le jugement part de l'abstrait, c'est-à-dire de la marque (top-down).

Ensuite, le déroulement de l'expérimentation, notamment le temps laissé pour comparer les objets entre eux, peut aussi inciter les participants à privilégier tel ou tel type de similarité. La pression temporelle peut favoriser le recours à une similarité relationnelle fondée sur un jugement de type holistique, plus affectif et réputé plus rapide que le recours à une similarité littérale plus sélective mais aussi plus lente à mettre en œuvre (Monga et John, 2010). La conformité à un schéma mental déjà établi, nourri par des expériences antérieures, et le fait que les réactions affectives de préférence, de plaisir ou de déplaisir précèdent toute autre forme 
d'évaluation cognitive (Zajonc, 1980), expliquent cet avantage de la similarité relationnelle. Etant holistique donc plus économe en ressources cognitives, le jugement de similarité relationnelle précède d'éventuelles évaluations plus analytiques (Fiske et al., 1987). Ceci se traduit inévitablement par des extensions de marques jugées plus familières, moins nouvelles et plus positivement évaluées (Estes et al., 2012).

Enfin, le choix des participants peut considérablement faire varier les résultats d'une expérimentation à l'autre. En effet, même si tout individu est capable de porter des jugements littéraux et relationnels, ses caractéristiques personnelles peuvent lui faire privilégier tel ou tel type de similarité. Les individus d'origine orientale (Asie et Méditerranée) sont ainsi plus enclins recourir à une similarité relationnelle car ils sont plus flexibles, plus interdépendants (Ahluwalia, 2008) et tolèrent mieux l'ambivalence (Thompson et Zanna, 1995). A contrario, en décontextualisant l'objet de son contexte, les cultures occidentales mettent davantage l'accent sur la similarité littérale. Des facteurs plus individuels peuvent également intervenir. Plus les consommateurs sont éduqués, experts, impliqués ou orientés vers un but, plus ils font appel à une similarité littérale (Hutchinson et Alba, 1991). Inversement, les hommes semblent avoir plus de capacité que les femmes à mobiliser la similarité relationnelle ; c'est pourquoi ils jugent plus favorablement qu'elles une extension dissimilaire réalisée par une marque fonctionnelle (Monga et Gürhan-Canli, 2012). La bonne humeur des individus au moment de l'exposition à l'extension favorise aussi les jugements de similarité relationnelle tandis que l'humeur maussade les rend plus analytiques donc plus critiques envers l'extension, ce qui encourage le recours à la similarité littérale (Sar, Duff et Anghelcev, 2011).

Si ces variables modératrices ne sont pas prises en compte, la contingence des résultats aux conditions de l'expérimentation rend délicate toute interprétation des recherches. Par exemple, 1'analyse par des professionnels de 54 extensions réelles de marques montre que la similarité littérale explique 16 à $17 \%$ de la variance de leur évaluation alors que la similarité relationnelle en explique 25\% (Estes et al., 2012). Ce résultat peut être interprété diversement : soit qu'il est plus facile d'étendre une marque en utilisant des associations abstraites (similarité relationnelle), notamment lorsque le nom de marque est typique, c'est à dire fortement associé à une catégorie (Heineken plutôt que Ralph Lauren : exemple donné par Batra, Lenk et Wedel 2010) ; soit que la plupart des extensions évaluées dans cette recherche concernaient des catégories de produits éloignées et des produits plus symboliques que fonctionnels; soit que la durée de l'évaluation était très restreinte ; soit tout simplement, que les évaluateurs étaient des hommes, d'origine orientale, peu experts, peu impliqués et en outre d'excellente humeur ! 


\section{CONCLUSION}

Alors que de nombreuses applications en marketing y font appel, le périmètre et les mécanismes de la similarité demeurent toujours aussi mystérieux (Medin, Goldstone et Gentner, 1993). Pour mieux utiliser ce concept dans les recherches à venir, ce travail avait pour but d'éclaircir deux points essentiels.

Le premier concerne la délimitation du concept de similarité par rapport à des concepts très proches. Une revue de la littérature en marketing et en psychologie met en évidence que la similarité n'est pas l'inverse exact de la différence, ce qui pose inévitablement des problèmes de mesure en termes de distance ou de proximité ${ }^{6}$. Ensuite, dans le processus de décision des consommateurs, la similarité se situe au niveau des croyances de base et non pas au niveau des attitudes ce qui la différencie du fit ; de plus, ce dernier est un transfert d'affect et non pas une comparaison. Enfin, dans sa conception littérale, elle ne permet de relier que des objets de même nature sur la base de caractéristiques concrètes alors que la congruence se fondant sur des relations plus abstraites, permet de relier des objets de natures très différentes. Ces distinctions conceptuelles qui complètent le travail de Maille et Fleck (2011) sur la congruence, devraient ainsi aider de futurs chercheurs à opter pour les concepts les mieux adaptés à leur problématique.

Le deuxième apport concerne l'existence de deux grands types de similarités, une similarité littérale et une similarité relationnelle, très proche voire confondue avec la congruence. La similarité littérale, historiquement la plus connue et la plus utilisée, repose sur un jugement de comparaison analytique des attributs ou des propriétés concrètes des objets en présence. Ceuxci sont jugés d'autant plus similaires qu'ils sont de même nature, c'est-à-dire qu'ils appartiennent à la même catégorie. Toutefois, ce type de similarité renforce systématiquement les différences (Medin, Goldstone et Gentner, 1990). En revanche, la similarité relationnelle (probablement de la congruence) permet de relier des objets de natures différentes, en se référant à un schéma établi et en activant une pensée analogique fondée sur des éléments extrinsèques ${ }^{7}$. Elle accentue les similarités (Gentner et Gunn, 2001) et favorise une évaluation plus rapide et souvent plus favorable des stimuli en présence.

Bien que cette distinction ne soit pas récente, peu de travaux en marketing en tirent toutes les conséquences méthodologiques ou managériales :

\footnotetext{
${ }^{6}$ Dans la théorie de la Gestalt, la loi de similarité stipule que notre cerveau regroupe les éléments qui paraissent semblables, c'est-à-dire qui partagent un certain nombre de propriétés. Elle diffère de la loi de la proximité pour laquelle le cerveau regroupe les éléments qui apparaissent souvent ensemble, dans une même zone perceptive.

${ }^{7}$ Pour de plus amples développements, voir Bezes (2010 a, 2010 b).
} 
- la plupart des échelles existantes, principalement dans le domaine de l'extension de marque, mixent ces deux conceptions plutôt que de les distinguer, ce qui réduit d'autant la compréhension du phénomène étudié. La mise au point relativement aisée d'échelles de mesure distinctes pour évaluer d'un côté, la similarité littérale et de l'autre, la similarité relationnelle comblerait donc un vide certain ;

- l'ensemble du dispositif d'une recherche peut être biaisé par le choix des objets présentés mais aussi par le profil des répondants ou par des instructions méthodologiques qui favorisent involontairement tel ou tel type de similarité. Ceci s'ajoute au biais lié au caractère asymétrique du concept de similarité qui conduit les répondants à choisir comme référent, le premier objet présenté ou celui qu'ils ont connu le plus tôt (Cunha et Laran, 2008);

- la similarité littérale et la similarité relationnelle s'appuient sur des processus cognitifs différents (comparaison analytique pour l'un et intégration holistique pour l'autre). Or, toute comparaison conduit à considérer les objets en présence comme des substituts relativement proches, ce qui met inévitablement l'accent sur des risques potentiels de cannibalisation. Par exemple, Collange (20058) montre qu'une marque de substitution similaire à la marque initiale bénéficie d'une image supérieure à celle de la marque initiale. Un lien entre similarité littérale et substituabilité est d'ailleurs constaté par Aurier (1993). Au contraire, l'intégration de ces mêmes objets dans un jugement d'ensemble (similarité relationnelle) permet au consommateur de penser ces objets comme complémentaires, ce qui met davantage l'accent sur de possibles fertilisations croisées (Estes et al., 2012). Mao et al. (2012) établissent eux aussi, un lien entre similarité littérale (relationnelle) et substitut (complément).

- Bien exploitée, cette distinction peut déboucher sur des applications extrêmement instructives dans le domaine des extensions de gammes ou de marques. La similarité littérale semble être ici plus pertinente pour étudier des extensions de marque ou plus encore de gamme, très associée aux attributs de son produit d'origine. Par exemple, les extensions basées sur « l'image fruitière »d'Andros sont de ce type (maîtrise du processus de préparation du fruit et déclinaison de cet univers dans des univers proches tels que les confitures, les produits laitiers et les boissons). Au contraire, la similarité relationnelle est plus apte à analyser les extensions de marques distantes : cas de Weight Watcher ou de Taillefine qui se développent sur différents marchés sans véritable rapport les uns avec les autres, en s'appuyant uniquement sur une image de marque centrée sur la diététique. Distinguer la similarité littérale et la similarité 
relationnelle peut donc permettre aux managers de piloter différemment les extensions de marques (Hem et Iversen, 2009), par exemple en réduisant la durée et la répétition des spots publicitaires des extensions relationnellement similaires (Estes et al., 2012).

Les recherches nouvelles devraient donc impérativement préciser le type de similarité mobilisé, les raisons de cette utilisation et les conséquences que ce choix conceptuel et méthodologique a sur les résultats obtenus. En effet, « si la définition et la mesure de la similarité sont ambiguës, le rôle de la similarité dans le transfert de connaissance et d'affect doit aussi être ambigu » (Martin et Stewart, 2001: 472). 
Figure 1. - Distinction conceptuelle entre la similitude, la similarité, la congruence et le fit

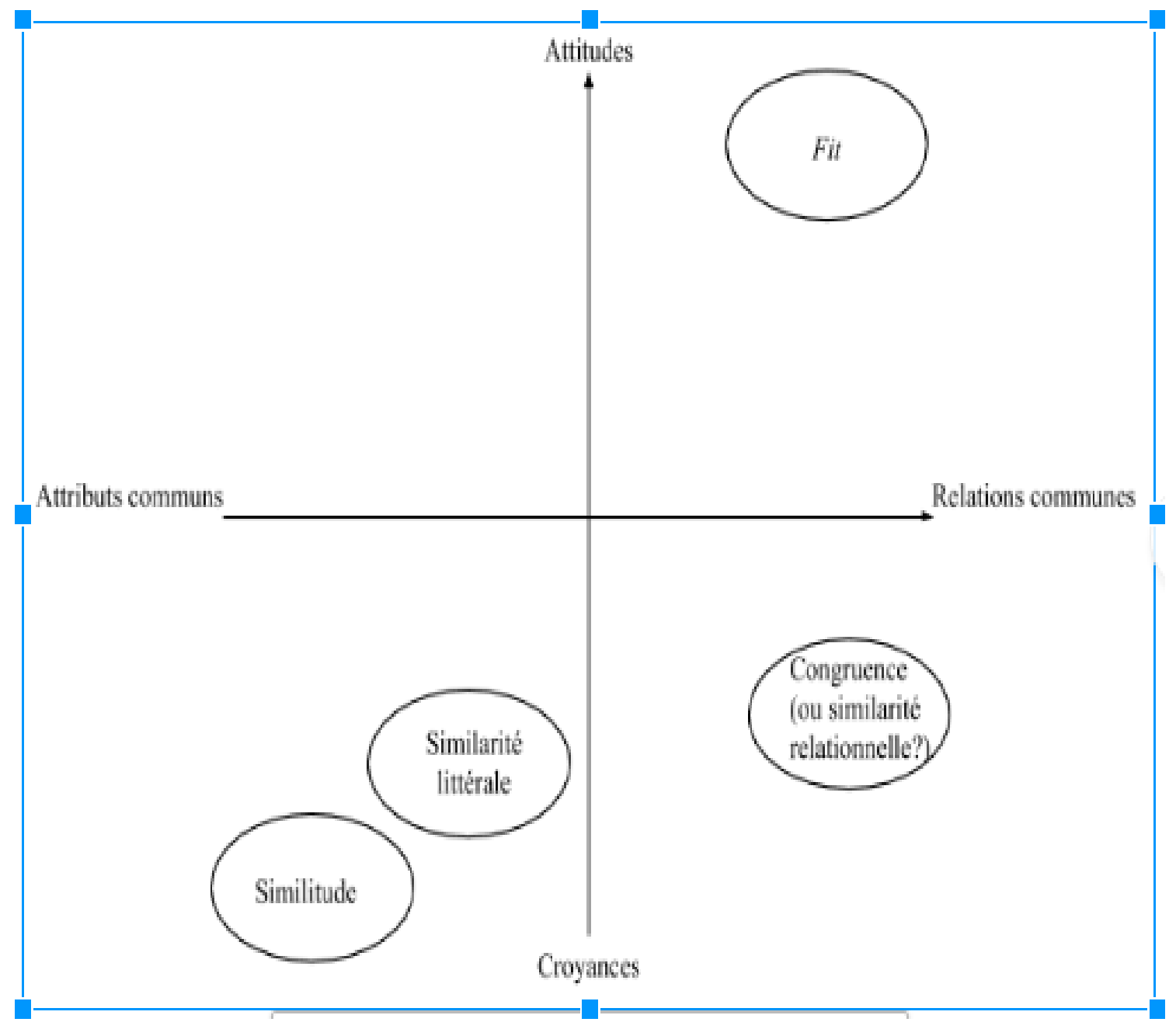


Tableau 1. - Panorama non exhaustif des principales mesures de la similarité par domaines d'application en marketing 


\begin{tabular}{|c|c|c|c|c|}
\hline Auteurs & Objet de recherche & Hypothèse principale & $\begin{array}{l}\text { Opérationnalisation de la } \\
\text { mesure }\end{array}$ & Limites \\
\hline \multicolumn{5}{|c|}{ Mesures de la similarité : l'approche sectorielle } \\
\hline Kim et Parkhe (2009) & $\begin{array}{l}\text { Analyse des alliances } \\
\text { d'entreprises. }\end{array}$ & $\begin{array}{l}\text { Les alliances entre firmes trop } \\
\text { similaires en termes de } \\
\text { ressources et de couverture des } \\
\text { marchés (similarité } \\
\text { compétitive) produisent un } \\
\text { résultat négatif à l'inverse des } \\
\text { alliances fondées sur une } \\
\text { similarité coopérative (même } \\
\text { socle culturel, même origine } \\
\text { nationale, même pratiques de } \\
\text { management). }\end{array}$ & $\begin{array}{l}\text { Echantillon de } 70 \text { firmes } \\
\text { américaines des industries } \\
\text { chimiques, électroniques ou } \\
\text { électriques. } \\
\text { Utilisation d'une échelle de } \\
\text { mesure multi-items pour } \\
\text { mesurer la similarité } \\
\text { compétitive et de plusieurs } \\
\text { construits pour mesurer la } \\
\text { similarité coopérative. }\end{array}$ & $\begin{array}{l}\text { Le fait d'avoir scindé en } 4 \\
\text { construits différents les } \\
\text { mesures de la similarité } \\
\text { coopérative, ne permet pas } \\
\text { véritablement une } \\
\text { comparaison avec le construit } \\
\text { de similarité compétitive qui } \\
\text { au contraire est totalement } \\
\text { agrégé. }\end{array}$ \\
\hline $\begin{array}{l}\text { Hofstede, Wedel et Steenkamp } \\
(2002)\end{array}$ & $\begin{array}{l}\text { Segmentation internationale } \\
\text { des marchés : application à } \\
\text { l'image des magasins de } \\
\text { viande en Europe. }\end{array}$ & $\begin{array}{l}\text { Les similarités spatiales entre } \\
\text { régions conditionnent les } \\
\text { similarités des perceptions et } \\
\text { besoins des consommateurs. }\end{array}$ & $\begin{array}{l}\text { Approche bayésienne } \\
\text { hiérarchique avec spécification } \\
\text { des niveaux de dépendance } \\
\text { spatiale ; test comparatif de } 4 \\
\text { modèles de prévision. }\end{array}$ & $\begin{array}{l}\text { Terrain réalisé sur l'Europe } \\
\text { avec des pays et régions qui } \\
\text { partagent une « culture } \\
\text { historique importante » et qui } \\
\text { peut ainsi avoir favorisé la } \\
\text { segmentation a priori. }\end{array}$ \\
\hline Grein (2000) & $\begin{array}{l}\text { Analyse des stratégies } \\
\text { marketing internationales des } \\
\text { constructeurs automobiles. }\end{array}$ & $\begin{array}{l}\text { Plus les pays sont similaires, } \\
\text { plus les stratégies marketing } \\
\text { des constructeurs le sont aussi. }\end{array}$ & $\begin{array}{l}\text { Calcul d'un score global } \\
\text { intégrant différents éléments } \\
\text { (PIB par tête, échanges } \\
\text { commerciaux entre pays,...) } \\
\text { puis de la distance euclidienne } \\
\text { pour chaque paire de pays } \\
\text { comparés. }\end{array}$ & $\begin{array}{l}\text { Prise en compte de variables } \\
\text { essentiellement } \\
\text { macroéconomiques. }\end{array}$ \\
\hline Creusen et Schoormans (1997) & $\begin{array}{l}\text { Analyse des effets sur les } \\
\text { préférences de différents types } \\
\text { de similarité. }\end{array}$ & $\begin{array}{l}\text { Les jugements de préférence } \\
\text { sont davantage influencés par } \\
\text { des bénéfices ou des attributs } \\
\text { d'image alors que les } \\
\text { jugements de similarité sont } \\
\text { davantage influencés par les } \\
\text { caractéristiques concrètes des } \\
\text { produits. }\end{array}$ & $\begin{array}{l}\text { Etude qualitative sur } 89 \\
\text { personnes et sur } 2 \text { types de } \\
\text { produits. }\end{array}$ & $\begin{array}{l}\text { Aucune comparaison des } \\
\text { effets de la similarité littérale } \\
\text { basée sur les attributs concrets } \\
\text { des objets et de la similarité } \\
\text { relationnelle basée sur les } \\
\text { attributs d'image et les } \\
\text { bénéfices. }\end{array}$ \\
\hline Green, Rao et Desarbo (1978) & $\begin{array}{l}\text { Définition des préférences à } \\
\text { l'égard de lieux de vacances. }\end{array}$ & $\begin{array}{l}\text { Utilisation de l'analyse } \\
\text { conjointe afin de déterminer } \\
\text { des groupes de préférence }\end{array}$ & $\begin{array}{l}\text { Classement de } 7 \text { lieux de } \\
\text { vacances (groupe 1). }\end{array}$ & $\begin{array}{l}\text { Limites en termes de notations } \\
\text { attribuées (validité inter- }\end{array}$ \\
\hline
\end{tabular}




\begin{tabular}{|c|c|c|c|c|}
\hline & & $\begin{array}{l}\text { fondés sur la similarité des } \\
\text { perceptions. }\end{array}$ & $\begin{array}{l}\text { Classement de } 6 \text { attributs en } \\
\text { trois niveaux selon leur } \\
\text { similarité perçue avec les } \\
\text { lieux (groupe } 2 \text { ). } \\
\text { Soumission de } 18 \text { profils de } \\
\text { lieux de vacances (groupe } 3 \text { ) } \\
\text { et utilisation de l'analyse } \\
\text { conjointe. }\end{array}$ & $\begin{array}{l}\text { juges), de validité externe et } \\
\text { de choix des attributs. }\end{array}$ \\
\hline Jain et Pinson (1976) & $\begin{array}{l}\text { Mise en évidence de } \\
\text { l'importance de l'ordre des } \\
\text { stimuli, de l'attention des } \\
\text { répondants et de l'implication } \\
\text { dans les jugements de } \\
\text { similarité. Les jugements de } \\
\text { similarité ont porté sur un } \\
\text { échantillon de } 8 \text { villes } \\
\text { américaines. }\end{array}$ & $\begin{array}{l}\text { L'ordre de présentation des } \\
\text { comparaisons par paires } \\
\text { produit des poids différents } \\
\text { pour chaque dimension. Par } \\
\text { ailleurs, augmenter le niveau } \\
\text { d'attention des individus } \\
\text { aboutit à une augmentation } \\
\text { artificielle du poids de chaque } \\
\text { dimension perçue. }\end{array}$ & $\begin{array}{l}\text { Utilisation du modèle Indscal } \\
\text { (individual difference scaling) } \\
\text { sur } 136 \text { répondants. Ce modèle } \\
\text { permet d'étudier les } \\
\text { différences entre juges. }\end{array}$ & $\begin{array}{l}\text { Pas de différence de jugement } \\
\text { de similarité pour aucune des } \\
\text { manipulations effectuées } \\
\text { (ordre, attention, implication). } \\
\text { La manière d'opérationnaliser } \\
\text { ces trois variables est discutée } \\
\text { et présentée comme peu } \\
\text { pertinente. }\end{array}$ \\
\hline Neidell (1972) & $\begin{array}{l}\text { Mesures de la similarité } \\
\text { appliquée au marché du } \\
\text { médicament. }\end{array}$ & $\begin{array}{l}\text { Utilisation de la comparaison } \\
\text { par paires afin de générer des } \\
\text { indices de similarité et } \\
\text { comparaison avec la méthode } \\
\text { dite de points d'ancrage. }\end{array}$ & $\begin{array}{l}\text { Expérimentation } 1 \text { : choix de } \\
\text { deux médicaments parmi trois } \\
\text { considérés comme les plus } \\
\text { similaires et de deux } \\
\text { considérés comme les moins } \\
\text { similaires entre eux } \\
\text { (sémantique différentielle). } \\
\text { Expérimentation } 2: \\
\text { soumission d'une échelle de } \\
\text { mesure par stimulus, notation } \\
\text { de chaque produit sur chaque } \\
\text { échelle (point d'ancrage). Les } \\
\text { stimuli sont générés par les } \\
\text { répondants. }\end{array}$ & $\begin{array}{l}\text { Difficulté de notations } \\
\text { concernant l'expérimentation } \\
\text { 1. L'expérimentation } 2 \text { est } \\
\text { plus riche en termes } \\
\text { d'indications sur les produits } \\
\text { mais ne fonctionne que pour } \\
\text { un nombre limité de stimuli } \\
(n=10) \text {. }\end{array}$ \\
\hline Fry et Claxton (1971) & $\begin{array}{l}\text { Mesure de la similarité de } 12 \\
\text { marques de cigarettes. }\end{array}$ & $\begin{array}{l}\text { Utilisation de l'analyse } \\
\text { sémantique différentielle et } \\
\text { des jugements directs de } \\
\text { similarité. Comparaison des } \\
\text { deux méthodes. }\end{array}$ & $\begin{array}{l}\text { Sélection de } 216 \text { fumeurs } \\
\text { londoniens. Mesure de la } \\
\text { similarité de } 12 \text { marques de } \\
\text { cigarettes, sélection de } 4 \\
\text { marques parmi les } 12 \text { jugées } \\
\text { les moins similaires en termes } \\
\text { d'image. Application d'une } \\
\text { analyse sémantique }\end{array}$ & $\begin{array}{l}\text { Congruence forte entre les } \\
\text { deux méthodes de mesure. } \\
\text { Méthodes jugées } \\
\text { complémentaires plutôt } \\
\text { qu'exclusives. Les limites de } \\
\text { l'analyse différentielle résident } \\
\text { dans l'estimation des } \\
\text { intervalles de mesure et dans }\end{array}$ \\
\hline
\end{tabular}




\begin{tabular}{|c|c|c|c|c|}
\hline & & & $\begin{array}{l}\text { différentielle concernant les } 4 \\
\text { marques choisies. } \\
\text { Utilisation du programme } \\
\text { Torsca d'analyse } \\
\text { multidimensionnelle. }\end{array}$ & $\begin{array}{l}\text { le risque d'omettre une } \\
\text { dimension. }\end{array}$ \\
\hline \multicolumn{5}{|c|}{ Comparaison des méthodes de mesure de la similarité } \\
\hline Johnson et Hudson (1996) & $\begin{array}{l}\text { Evaluation de la perception } \\
\text { des utilisateurs des méthodes } \\
\text { d'analyse des marchés. Deux } \\
\text { méthodes sont évaluées : les } \\
\text { méthodes dites spatiales } \\
\text { permettant d'évaluer la } \\
\text { similarité des marques et des } \\
\text { produits sur une carte et les } \\
\text { méthodes dites de clustering } \\
\text { ou classification. }\end{array}$ & $\begin{array}{l}\text { La représentation spatiale de la } \\
\text { similarité est plus intuitive et } \\
\text { mieux assimilée par les } \\
\text { managers que la représentation } \\
\text { par classification. } \\
\text { Les deux méthodes seraient } \\
\text { complémentaires dans leur } \\
\text { approche de la similarité. La } \\
\text { représentation spatiale } \\
\text { permettrait d'évaluer la } \\
\text { concurrence entre marques de } \\
\text { la même catégorie alors que } \\
\text { les méthodes de classification } \\
\text { apportent un éclairage sur la } \\
\text { concurrence entre catégories } \\
\text { elles-mêmes. }\end{array}$ & $\begin{array}{l}\text { Phase } 1 \text { : les sujets ont classé } \\
\text { par paires six stimuli, chacun } \\
\text { correspondant à } 12 \text { produits ou } \\
\text { services. } \\
\text { Phase } 2: \text { différentes cartes et } \\
\text { classifications ont été } \\
\text { élaborées. Une échelle de } \\
\text { mesure de l'utilité perçue des } \\
\text { deux méthodes a été soumise à } \\
\text { un deuxième échantillon. } \\
\text { Les résultats montrent que les } \\
\text { deux types d'analyse sont } \\
\text { perçus comme plus utiles dans } \\
\text { le cas de la concurrence entre } \\
\text { marques qu'entre catégories. } \\
\text { Par ailleurs, la familiarité } \\
\text { préalable avec les marques ou } \\
\text { catégories analysées ont une } \\
\text { influence sur la perception de } \\
\text { l'utilité des deux types } \\
\text { d'études. }\end{array}$ & $\begin{array}{l}\text { Limitation des résultats aux } \\
\text { analyses concurrentielles. } \\
\text { Stimuli choisis parfois trop } \\
\text { protypiques ce qui confère aux } \\
\text { solutions fondées sur la } \\
\text { catégorisation un avantage sur } \\
\text { les méthodes spatiales. }\end{array}$ \\
\hline Wilkes et Wilcox (1977) & $\begin{array}{l}\text { Réflexion sur la validité et } \\
\text { fiabilité des jugements de } \\
\text { similarité. Commentaires } \\
\text { autour d'une recherche faite } \\
\text { par Summers et MacKay } \\
\text { (1976) sur le marché } \\
\text { automobile et les } \\
\text { emplacements de campus. }\end{array}$ & $\begin{array}{l}\text { Mesure via les jugements } \\
\text { directs de similarité } \\
\text { (comparaison de paires). }\end{array}$ & $\begin{array}{l}\text { Deux échantillons d'individus } \\
\text { ont noté deux types de stimuli } \\
\text { (automobile et emplacement } \\
\text { de campus) Les données ont } \\
\text { été triangulisées, agrégées puis } \\
\text { retestées sur un deuxième } \\
\text { groupe un mois plus tard. } \\
\text { Trois cartes perceptuelles ont } \\
\text { été soumises deux semaines } \\
\text { après (carte données }\end{array}$ & $\begin{array}{l}\text { Dans l'expérimentation de } \\
\text { Summers et MacKay (1976), } \\
\text { les sujets s'étaient révélés } \\
\text { incapables d'identifier leur } \\
\text { propre mapping. Les } \\
\text { jugements directs de similarité } \\
\text { ne sont pas une mesure valide } \\
\text { des perceptions. } \\
\text { La duplication de Wilkes et } \\
\text { Wilcox (1977) sur des }\end{array}$ \\
\hline
\end{tabular}




\begin{tabular}{|c|c|c|c|c|}
\hline & & & $\begin{array}{l}\text { individuelles et agrégées). } \\
\text { Pour chaque carte, les sujets } \\
\text { notaient la carte sur une } \\
\text { échelle à } 7 \text { modalités (ne } \\
\text { correspond pas à ma } \\
\text { perception ou reflète ma } \\
\text { perception). }\end{array}$ & $\begin{array}{l}\text { étudiants aboutit à une } \\
\text { conclusion différente en } \\
\text { effectuant des rotations des } \\
\text { mappings (rotation de } 90^{\circ} \text { par } \\
\text { rapport à la source). } \\
\text { Néanmoins, les auteurs } \\
\text { insistent sur la difficulté des } \\
\text { consommateurs à faire un lien } \\
\text { entre une carte perceptuelle et } \\
\text { une combinaison par paires. }\end{array}$ \\
\hline Lehmann (1972) & $\begin{array}{l}\text { Mise en place d'une procédure } \\
\text { d'élaboration d'un mapping } \\
\text { perceptuel fondé sur les } \\
\text { préférences. Utilisation d'une } \\
\text { matrice de transition comme } \\
\text { matrice de similarité. } \\
\text { Etude de l'influence de la } \\
\text { similarité perçue des marques } \\
\text { dans les cas d'abandon d'une } \\
\text { marque pour une autre. }\end{array}$ & $\begin{array}{l}\text { La similarité entre deux } \\
\text { marques peut être vue comme } \\
\text { la moyenne des probabilités } \\
\text { d'abandon d'une marque pour } \\
\text { une autre. }\end{array}$ & $\begin{array}{l}\text { L'utilisation du programme } \\
\text { multidimensionnel Torsca a } \\
\text { permis d'établir une carte } \\
\text { perceptuelle, base de } \\
\text { l'élaboration de la matrice de } \\
\text { transition. } 8 \text { soft drinks ont été } \\
\text { proposés à } 264 \text { personnes } \\
\text { durant une période d'achat de } \\
12 \text { mois. Les abandons et les } \\
\text { changements de marque ont } \\
\text { été mesurés (matrice de } \\
\text { transition) et comparés aux } \\
\text { différentes mesures effectuées } \\
\text { de similarité. }\end{array}$ & $\begin{array}{l}\text { Difficulté d'utiliser les } \\
\text { matrices de transition comme } \\
\text { matrice de similarité : } \\
\text { insuffisance d'homogénéité } \\
\text { des individus étudiés, } \\
\text { problème de détermination des } \\
\text { périodes d'achat, multi- } \\
\text { dimensionnalité des critères de } \\
\text { choix et de leur poids dans } \\
\text { l'achat, recherche de variété... }\end{array}$ \\
\hline \multicolumn{5}{|c|}{ Mesures de la similarité interpersonnelle } \\
\hline $\begin{array}{l}\text { Bourcier-Bequaert et De } \\
\text { Barnier (2014) }\end{array}$ & $\begin{array}{l}\text { Effets de la similarité d'âge } \\
\text { subjectif sur la marque } \\
\text { présente dans la publicité. }\end{array}$ & $\begin{array}{l}\text { La similarité d'âge subjectif } \\
\text { du mannequin et du récepteur } \\
\text { agit directement sur la } \\
\text { similarité globale (source- } \\
\text { récepteur) et indirectement sur } \\
\text { l'attitude à l'égard de la } \\
\text { marque et sur la considération } \\
\text { d'achat. }\end{array}$ & $\begin{array}{l}\text { Test de deux annonces avec } \\
\text { deux mannequins d'âges } \\
\text { différents, sur } 221 \text { femmes } \\
\text { âgées de } 60 \text { à } 70 \text { ans } \\
\text { Utilisation des échelles de } \\
\text { mesure directe de Guiot } \\
\text { (2001), Chang (2008) et } \\
\text { McKirnan } \text { et al. (1983). }\end{array}$ & $\begin{array}{l}\text { N'ont été étudiés que les effets } \\
\text { de mannequin jeune et âgé sur } \\
\text { des femmes seniors. Nécessité } \\
\text { de tester les effets d'un } \\
\text { mannequin entre deux âges et } \\
\text { de prendre en compte un } \\
\text { échantillon plus large en } \\
\text { termes d'âge et de genre. }\end{array}$ \\
\hline David-Ignatieff (2012) & $\begin{array}{l}\text { Similarité perçue à l'égard } \\
\text { d'un avatar : élaboration d'une } \\
\text { échelle de mesure définissant } \\
\text { l'homophilie comme le degré } \\
\text { de similarité des agents qui } \\
\text { interagissent. }\end{array}$ & $\begin{array}{l}\text { Echelle sémantique qui } \\
\text { mesure un construit } \\
\text { multidimensionnel fondé sur } 4 \\
\text { types de similarité : la } \\
\text { similarité d'attitude, la } \\
\text { similarité d'éducation, la }\end{array}$ & $\begin{array}{l}\text { Utilisation de deux dimensions } \\
\text { de l'échelle à savoir la } \\
\text { dimension d'attitude et la } \\
\text { dimension d'apparence (items, } \\
\text { l'avatar est très similaire à } \\
\text { moi, me ressemble }\end{array}$ & $\begin{array}{l}\text { Difficulté à confirmer la } \\
\text { dimension similarité des } \\
\text { valeurs (l'avatar pense comme } \\
\text { moi). Une seule dimension est } \\
\text { conservée dans l'analyse }\end{array}$ \\
\hline
\end{tabular}




\begin{tabular}{|c|c|c|c|c|}
\hline & & $\begin{array}{l}\text { similarité des valeurs, la } \\
\text { similarité d'apparence. }\end{array}$ & $\begin{array}{l}\text { physiquement, a une } \\
\text { apparence comme la mienne, } \\
\text { pense beaucoup comme moi). } \\
\text { L'échelle a été testée sur } 320 \\
\text { participants soumis à un site } \\
\text { Internet avec différentes } \\
\text { versions d'avatars. }\end{array}$ & $\begin{array}{l}\text { confirmatoire (la similarité } \\
\text { d'apparence). }\end{array}$ \\
\hline \multicolumn{5}{|c|}{ Mesures de la similarité appliquée aux recherches cross-culturelles } \\
\hline $\begin{array}{l}\text { Watchravesringkan, Yan et } \\
\text { Yurchisin (2008) }\end{array}$ & $\begin{array}{l}\text { Etude cross-culturelle de la } \\
\text { perception des prix pour } \\
\text { quatre pays du sud-est } \\
\text { asiatique : Chine, Corée du } \\
\text { Sud, Taiwan et Thailande. }\end{array}$ & $\begin{array}{l}\text { Utilisation de l'analyse } \\
\text { factorielle confirmatoire } \\
\text { multigroupes afin d'examiner } \\
\text { le degré de similarité des } \\
\text { perceptions du prix dans un } \\
\text { contexte multiculturel. }\end{array}$ & $\begin{array}{l}\text { Sur un échantillon de } 950 \\
\text { étudiants, mise en évidence de } \\
\text { cinq dimensions constitutives } \\
\text { de la perception du prix : } \\
\text { perception du rapport qualité- } \\
\text { prix, sensibilité au prestige, } \\
\text { conscience de la valeur, } \\
\text { propension à la vente, } \\
\text { expertise-prix. }\end{array}$ & $\begin{array}{l}\text { Utilisation d'un échantillon de } \\
\text { convenance rendant les } \\
\text { résultats peu généralisables. }\end{array}$ \\
\hline $\begin{array}{l}\text { Van Auken, Barry et Bagozzi } \\
\text { (2006) }\end{array}$ & $\begin{array}{l}\text { Etude cross-culturelle de l'âge } \\
\text { cognitif afin de dégager des } \\
\text { similarités entre les différentes } \\
\text { dimensions du construit } \\
\text { (Etats-Unis par opposition au } \\
\text { Japon). }\end{array}$ & $\begin{array}{l}\text { Test de différentes échelles de } \\
\text { mesure : une échelle } \\
\text { sémantique différentielle, une } \\
\text { échelle de Likert et une } \\
\text { échelle de ratio. }\end{array}$ & $\begin{array}{l}\text { Analyse effectuée sur deux } \\
\text { échantillons de seniors } \\
\text { japonais et américains. La } \\
\text { variance la plus forte a été } \\
\text { observée pour l'échelle } \\
\text { sémantique différentielle sur } \\
\text { l'ensemble des dimensions du } \\
\text { construit : âge cognitif, âge } \\
\text { idéal, âge le moins désiré. } \\
\text { L'échelle de ratio met en } \\
\text { évidence plus de similarité } \\
\text { entre répondants que l'échelle } \\
\text { sémantique différentielle ou } \\
\text { l'échelle de Likert. } \\
\text { Les plus fortes similarités ont } \\
\text { été dégagées entre seniors } \\
\text { japonais masculins et } \\
\text { féminins, les plus fortes } \\
\text { différences entre Japonais et } \\
\text { Américains. }\end{array}$ & $\begin{array}{l}\text { Manipulation délicate des } \\
\text { mesures du fait du caractère } \\
\text { considéré comme abstrait de } \\
\text { certaines notations pour les } \\
\text { Japonais. }\end{array}$ \\
\hline
\end{tabular}




\begin{tabular}{|c|c|c|c|c|}
\hline Völckner et Sattler (2006) & $\begin{array}{l}\text { Analyse de } 10 \text { facteurs qui } \\
\text { expliquent la réussite d'une } \\
\text { extension de marque. }\end{array}$ & & $\begin{array}{l}5 \text { extensions réelles de } 25 \\
\text { marques différentes prises en } \\
\text { compte, évaluées par } 2426 \\
\text { étudiants. } \\
\text { La similarité globale entre la } \\
\text { marque-mère et son extensions } \\
\text { est intégrée dans l'échelle de } \\
\text { mesure du fit, au même titre } \\
\text { que la capacité de la marque à } \\
\text { réaliser l'extension. }\end{array}$ & $\begin{array}{l}\text { Interrogation de la part de } \\
\text { Völckner et Sattler sur ce qui } \\
\text { constitue vraiment le fit. }\end{array}$ \\
\hline Klink et Smith (2001) & $\begin{array}{l}\text { Analyse des facteurs qui } \\
\text { modèrent la relation entre la } \\
\text { similarité globale (fit) et } \\
\text { l'évaluation de l'extension de } \\
\text { marque. }\end{array}$ & $\begin{array}{l}\text { Les facteurs qui modèrent la } \\
\text { relation entre la similarité } \\
\text { globale et l'évaluation de } \\
\text { l'extension de marque. }\end{array}$ & $\begin{array}{l}\text { La similarité globale entre la } \\
\text { marque mère et la catégorie en } \\
\text { extension (le fit pour ces } \\
\text { auteurs) est mesurée par un } \\
\text { instrument de mesure qui } \\
\text { regroupe tout à la fois des items } \\
\text { décrivant une similarité } \\
\text { littérale (caractéristiques et } \\
\text { fonctions du produit, processus } \\
\text { de fabrication) et des items } \\
\text { décrivant une similarité } \\
\text { relationnelle (besoins satisfaits, } \\
\text { situations d'usage). }\end{array}$ & $\begin{array}{l}\text { Limite méthodologique: le } \\
\text { construit de similarité globale } \\
\text { est en fait formatif (et non } \\
\text { réflectif) puisqu'il est constitué } \\
\text { de deux types de similarité. Il } \\
\text { devrait donc être dissocié ou } \\
\text { validé comme un construit } \\
\text { formatif. Nécessité de } \\
\text { dupliquer l'analyse avec la } \\
\text { conception du fit de } \\
\text { Broniarczyk et Alba (1994). }\end{array}$ \\
\hline Dacin et Smith (1994) & $\begin{array}{l}\text { Nombre possible, qualité et } \\
\text { liaisons des extensions avec la } \\
\text { marque-mère qui ne fragilisent } \\
\text { pas la marque-mère. }\end{array}$ & $\begin{array}{l}\text { Le manque de cohérence dans } \\
\text { le portefeuille de produits de la } \\
\text { marque réduit l'appréciation de } \\
\text { la qualité de l'extension. }\end{array}$ & $\begin{array}{l}\text { Similarité entre les produits de } \\
\text { marque-mère et ceux de son } \\
\text { extension mesurée par } 3 \text { items } \\
\text { (mêmes types de besoins } \\
\text { satisfaits, mêmes situations } \\
\text { d'utilisation, caractéristiques } \\
\text { physiques des produits). }\end{array}$ & $\begin{array}{l}\text { Extension fictive décrite trop } \\
\text { succinctement. }\end{array}$ \\
\hline Smith et Park (1992) & $\begin{array}{l}\text { Analyse des effets des } \\
\text { extensions de marques sur la } \\
\text { part de marché et l'efficacité } \\
\text { publicitaire. }\end{array}$ & $\begin{array}{l}\text { La forte similarité entre le } \\
\text { produit et les autres produits } \\
\text { référents de la marque } \\
\text { maximisent les effets de } \\
\text { l'extension. }\end{array}$ & $\begin{array}{l}\text { Analyse factorielle de la } \\
\text { similarité qui permet de } \\
\text { distinguer une similarité } \\
\text { intrinsèque (littérale) et une } \\
\text { similarité extrinsèque } \\
\text { (relationnelle). }\end{array}$ & $\begin{array}{l}\text { Les extensions analysées étant } \\
\text { des extensions réussies, les } \\
\text { scores de force de la marque et } \\
\text { de similarité sont trop élevés } \\
\text { pour évaluer si des scores plus } \\
\text { faibles diminueraient l'effet } \\
\text { sur la part de marché. }\end{array}$ \\
\hline
\end{tabular}




\begin{tabular}{|c|c|c|c|c|}
\hline $\begin{array}{l}\text { Park, Milberg et Lawson } \\
\text { (1991) }\end{array}$ & $\begin{array}{l}\text { Analyse des effets de la } \\
\text { similarité littérale et de la } \\
\text { similarité relationnelle sur } \\
\text { l'évaluation des extensions de } \\
\text { marques. }\end{array}$ & $\begin{array}{l}\text { L'extension d'une marque } \\
\text { fonctionnelle est davantage } \\
\text { favorisée par une similarité } \\
\text { basée sur les caractéristiques } \\
\text { des produits alors qu'une } \\
\text { extension de prestige est } \\
\text { favorisée basée sur des } \\
\text { éléments plus abstraits. }\end{array}$ & $\begin{array}{l}\text { Evaluation par } 30 \text { individus de } \\
\text { la similarité entre deux } \\
\text { marques de montres (Timex et } \\
\text { Rolex) et leurs extensions. } \\
\text { Codage des réponses en } \\
\text { aveugle par deux codeurs qui } \\
\text { isolent une similarité littérale } \\
\text { (caractéristiques des produits, } \\
\text { usage et fonctions des } \\
\text { produits) et une similarité plus } \\
\text { relationnelle (catégories } \\
\text { fonctionnelles ou } \\
\text { prestigieuses). }\end{array}$ & 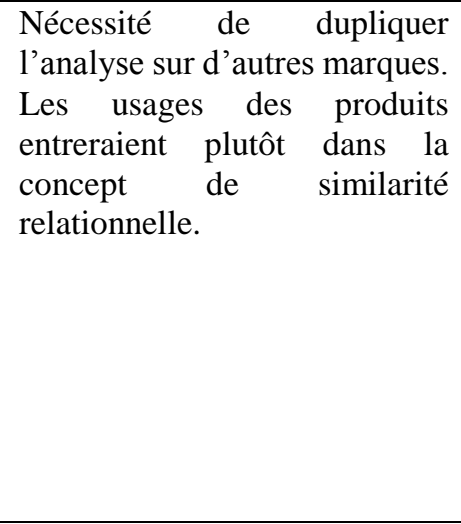 \\
\hline
\end{tabular}




\section{RÉFÉRENCES BIBLIOGRAPHIQUES}

Aaker D et Keller K (1990) Consumer evaluations of brand extensions. Journal of Marketing 54 (1): 27-41.

Alba J et Hutchinson W (1987) Dimensions of consumer expertise. Journal of Consumer Research 13(4): 411-454.

Ahluwalia R (2008) How far can a brand stretch? Understanding the role of self-construal. Journal of Marketing Research 337(45): 337-350.

Aurier P (1993) Analyse de la structure des marchés. Le point sur les modèles. Recherche et Applications en Marketing 8(2): 79-104.

Barsalou L (1983) Ad hoc categories. Memory \& Cognition 11(3): 211-227.

Barsalou L (1985) Ideals, central tendency, and frequency of instantiation as determinants of graded structure in categories. Journal of Experimental Psychology: Learning, Memory, and Cognition 11(4): 629-654.

Bassok M et Medin D (1997) Birds of a Feather Flock Together: Similarity Judgments with Semantically Rich Stimuli. Journal of Memory and Language 36 (3): 311-336.

Batra R, Lenk P et Wedel M (2010) Brand extension strategy planning: empirical estimation of brand-category personality fit and atypicality. Journal of Marketing Research 47(2): 335-347.

Bèzes C (2010) Tout ce qui est congruent, est-il similaire ? Propositions de définition du concept de congruence en marketing. In : 26ème Congrès International de l'AFM, Le Mans, France, Mai 2010.

Bèzes C (2013) Effect of channel congruence on a retailer's image. International Journal of Retail \& Distribution Management 41(4): 254-273.

Bèzes C et Dubois P-L (2013) A conceptual and empirical distinction between the concepts of congruence and fit: the example of retailer extensions. In La Londe Consumer Behavior Conference, La Londe les Maures, France, Juin 2013.

Bourcier-Bequaert B et De Barnier V (2014) Les effets de l'âge du mannequin sur les consommateurs seniors et sur la marque présente dans la publicité : le rôle de la similarité. Décisions Marketing 76: 77-92.

Boush D (1997) Brand name effects on interproduct similarity judgments. Marketing Letters 8(4): 419-427. 
Boush D, Shipp S, Loken B, Gencturk E, Crockett S, Kennedy E, Minshall B, Misurell D, Rochford L et Strobel J (1987) Affect generalization to similar and dissimilar brand extensions. Psychology \& Marketing 4(3): 225-237.

Bridges S (1989) A schema unification model of brand extensions. Advances in Consumer Research 19(1): 257-258.

Bridges S Keller K et Sood S (2000) Communication strategies for brand extensions: enhancing perceived fit by establishing explanatory links. Journal of Advertising 39(4) : 1-11. Broniarczyk S et Alba J (1994) The importance of the brand in brand extension. Journal of Marketing Research 31(2): 214-228.

Brudvig S et Raman P (2006) The impact of fit and goal congruency on consumers' attitude to brand extensions. Marketing Management Journal 16 (2): 171-178.

Bruner J et Postman L (1949) On the perception of incongruity: a paradigm. Journal of Personality 18(2): 206-223.

Byrne D et Griffitt W (1973) Interpersonal attraction. Annual Review of Psychology 24(1): 317336.

Byrne D, Griffitt W et Stefaniak D (1967) Attraction and similarity of personality characteristics. Journal of Personality and social Psychology 5(1): 82-90.

Cech C (1989) Congruity and the expectancy hypothesis. Journal of Experimental Psychology: Learning, Memory, and Cognition 15(6): 1129-1133.

Cegarra J-J et Merunka D (1993) Les extensions de marque : concepts et modèles. Recherche et Applications en Marketing 8(1): 53-76.

Chandon J-L et Strazziéri A (1986) Une analyse de structure de marché sur la base de la mesure de l'ensemble évoqué. Recherche et Applications en Marketing 1(1): 17-39.

Corfman K (1991) Comparability and comparison levels used in choices among consumer products. Journal of Marketing Research 28( 3): 368-374.

Cunha M et Laran J (2008) Asymmetries in the sequential learning of brand associations: Implications for the early entrant advantage. Journal of Consumer Research 35 (5): 788-799. Cohen J et Basu K (1987) Alternative models of categorization: toward a contingent processing framework. Journal of Consumer Research 13(4): 455-472.

Collange V (2005) L'impact du changement de nom de marque sur l'attitude vis-à-vis du produit. Thèse de Doctorat en Sciences de Gestion, Université Paul Cézanne Aix-Marseille 3. Creusen M et Schoormans J (1997) The nature of differences between similarity and preference judgements: A replication with extension. International Journal of Research in Marketing 14(1): 81-87. 
Czellar S (2003) Consumer attitude toward brand extensions: an integrative model and research propositions. International Journal of Research in Marketing 20: 97-115.

Dacin P et Smith D (1994) The effect of brand portfolio characteristics on consumer evaluations of brand extensions. Journal of Marketing Research 31( 2): 229-242.

Damak L (1997) Corps du consommateur et design du produit: recherche d'une similarité ou d'une complémentarité? In Cahier de Recherche 257, Centre de Recherche DMSP, Paris Dauphine.

David-Ignatieff A (2012) Détermination de l'âge idéal de l'avatar en fonction de l'âge de l'internaute cible du site Internet. Thèse de Doctorat, HEC Montréal, Canada.

Derbaix C et Sjöberg L (1994) Movie stars in space: A comparison of preference and similarity judgments. International Journal of Research in Marketing 11(3): 261-274. Dimitriadis S (1993) L'extension des marques et des enseignes : cadre conceptuel et problématique managériale. Recherche et Applications en Marketing 8(3): 21-44.

Douglas S et Craig S (1996) Executive insights: global portfolio planning and market interconnectedness. Journal of International Marketing 4(1): 93-110.

Dröge C et Darmon R (1987) Associative positioning strategies through comparative advertising: Attribute versus overall similarity approaches. Journal of Marketing Research 24(4): 377-388.

Dubé L et Schmitt B (1999) The effect of a similarity versus dissimilarity focus in positioning strategy: the moderating role of consumer familiarity and product category. Psychology \& Marketing 16 (3): 211-224.

Estes Z, Gibbert M, Guest D et Mazursky D (2012) A dual-process model of brand extension: taxonomic feature-based and thematic relation-based similarity independently drive brand extension evaluation. Journal of Consumer Psychology 22( 1): 86-101.

Fiske S, Neuberg S, Beattie A et Milberg S (1987) Category-based and attribute-based reactions to others: some informational conditions of stereotyping and individuating process. Journal of Experimental Social Psychology 23( 5): 399-427.

Fry J et Claxton J (1971) Semantic differential and nonmetric multidimensional scaling descriptions of brand images. Journal of Marketing Research 8( 2) : 238-240.

Galan J-P (2009) Musique et réponses à la publicité : effets des caractéristiques, de l'appréciation et de la congruence musicales. Recherche et Applications en Marketing 24 (4) : $3-21$.

Gefen D et Straub D (1997) Gender differences in the perception and use of e-mail: An extension to the Technology Acceptance Model. MIS Quarterly 21( 4): 389-400. 
Gentner D (1988) Metaphor as structure mapping: the relational shift. Child Development 59: 47-59.

Gentner D et Gunn V (2001) Structural alignment facilitates the noticing of differences. Memory \& Cognition 29(4): 565-577.

Gentner D et Markman A (1994) Structural alignment in comparison: no difference without similarity. Psychological Science 5(3): 152-158.

Gentner D et Markman A (1997) Structure mapping in analogy and similarity. American Psychologist 52( 1): 45-56.

Gill T et Dubé L (2007) What is a leather iron or a bird phone? Using conceptual combinations to generate and understand new product concepts. Journal of Consumer Psychology 17( 3): 202-217.

Goldstone R, Medin D et Gentner D (1991) Relational similarity and the non-independence of features in similarity judgments. Cognitive Psychology 23( 2): 222-262.

Goodman N (1972) Seven strictures on similarity. Problems and Projects. New-York, BobsMerill.

Green P, Rao V et Desarbo W (1978) Incorporating group-level similarity judgments in conjoint analysis. Journal of Consumer Research 5(3): 187-193.

Gregan-Paxton J et John R (1997) Consumer learning by analogy: a model of internal knowledge transfer. Journal of Consumer Research 24 ( 3) : 266-284.

Grein A (2000) The impact of market similarity on international marketing strategies: The automobile industry in western Europe. International Business Review 42( 2): 167-186.

Hassanein K et Head M (2006) The impact of infusing social presence in the web interface: An investigation across product types. International Journal of Electronic Commerce 10(2): 3155.

Heckler S et Childers T (1992) The role of expectancy and relevancy in memory for verbal and visual information: what is incongruency? Journal of Consumer Research 18: 475-492.

Hem L et Iversen N (2002) Decomposed similarity measures in brand extensions. Advances in Consumer Research 29: 199-206.

Hem L et Iversen N (2009) Effects of different types of perceived similarity and subjective knowledge in evaluations of brand extensions. International Journal of Market Research 51( 6): 797-818.

Herr P, Farquhar P et Fazio R (1996) Impact of dominance and relatedness on brand extensions. Journal of Consumer Psychology 5 ( 2): 135-159. 
Hirschmann E (1980) Innovativeness, novelty seeking, and consumer creativity. Journal of Consumer Research 7(3): 283-295.

Hofstede F, Wedel M et Steenkamp J-B (2002) Identifying spatial segments in international markets. Marketing Science 21(2): 160-77.

Hollingworth H (1913) Judgments of similarity and difference. Psychological Review 20(4): 271-289.

Hutchinson J et Alba J (1991) Ignoring irrelevant information: situational determinants of consumer learning. Journal of Consumer Research 18 (3): 325-345.

Jain A et Pinson C (1976) The effect of order of presentation of similarity judgments on multidimensional scaling results: an empirical examination. Journal of Marketing Research 13(4): 435-439.

Jiang L, Hoegg J, Dahl D et Chattopadhyay A (2010) The persuasive role of incidental similarity on attitudes and purchase intentions in a sales context. Journal of Consumer Research 36(5): 778-791.

Johnson M (1981) Context effects in product perception. Advances in Consumer Research, 8(1): 112-115.

Johnson M (1984) Consumer choice strategies for comparing non comparable alternatives. Journal of Consumer Research 11( 3): 741-753.

Johnson M et Horne D (1987) Subject/referent positioning in comparative advertising: a pilot study. Advances in Consumer Research 14 (1): 164-167.

Johnson M et Horne D (1988) The contrast model of similarity and comparative advertising. Psychology \& Marketing 5( 3) : 211-232.

Johnson M et Hudson E (1996) On the perceived usefulness of scaling techniques in market analysis. Psychology \& Marketing 13(7): 653-675.

Kamins M et Gupta K (1984) Congruence between spokesperson and product type: a matchup hypothesis perspective. Psychology \& Marketing 11(6): 569-586.

Kellaris J, Cox A et Cox D (1993) The effect of background music on ad processing: a contingency explanation. Journal of Marketing 57: 114-125.

Keller K et Aaker D (1992) The effects of sequential introduction of brand extensions. Journal of Marketing Research 29(1): 35-50.

Khan M et Paivio A (1988) Memory for schematic and categorical information: a replication and extension of Rabinowitz and Mandler. Journal of Experimental Psychology: Learning, Memory and Cognition 14(3): 558-561. 
Kim J et Parkhe A (2009) Competing and cooperating similarity in global strategic alliances: An exploratory examination. British Journal of Management 20(3): 363-376.

Kirmani A et Shiv B (1998) Effects of source congruity on brand attitudes and beliefs: the moderating role of issue-relevant elaboration. Journal of Consumer Psychology 7 (1): 25-47. Klink R et Smith D (2001) Threats to the external validity of brand extension research. Journal of Marketing Research 38 (3): 326-335.

Kruhmans C (1978) Concerning the applicability of geometric models to similarity data: The interrelationship between similarity and spatial density. Psychological Review 85( 5): 445-163. Kwon H et Ha S (2013) The effects of perceived similarity with others on shopping mall satisfaction. Society for Marketing Advances Proceedings 25: 249-250.

Law S (2002) Can repeating a brand claim lead to memory confusion? The effects of claim similarity and concurrent repetition. Journal of Marketing Research 39 (3): 366-378.

Leibniz G (1995) La caractéristique géométrique. traduit par M. Parmentier, Vrin.

Lehmann D (1972) Judged similarity and brand-switching data as similarity measures. 9(3): 331-334.

Lemoine J-F et Chérif E (2012) Comment générer de la confiance envers un agent virtuel à l'aide de ses caractéristiques? Une étude exploratoire. Management \& Avenir 58: 169-188.

Loken B (2006) Consumer psychology: categorization, inferences, affect, and persuasion. Annual Review of Psychology 57 : 453-485.

Maille V (2007), L'intensité du goût et de la couleur de produits alimentaires : l'influence de l'incongruence perçue sur l'acceptabilité. Actes du $23^{\text {ème }}$ Congrès International de l'AFM, Aixles-Bains, France, Mai 2007, pp.1-25.

Maille V et Fleck N (2011) Congruence perçue par le consommateur : vers une clarification du concept, de sa formation et de sa mesure. Recherche et Applications en Marketing 26 (2) : 77 111.

Mandler G (1982) The structure of value: accounting for taste. In: M.S. Clark et S.T. Fiske (cood), Affect and Cognition: Annual Carnegie Symposium, 17, Hillsdale, Lawrence Erlbaum Associates, pp. 3-36.

Mao H, Mariadoss B, Echambadi R et Chennamaneni P (2012) Brand extensions via complements or substitutes: The moderating role of manufacturing transferability. Marketing Letters 23 (1): 279-292.

Markman A et Wisniewski E (1997) Similar and different: the differentiation of basic-level categories. Journal of Experimental Psychology: Learning, Memory, and Cognition 23 (1): 5470. 
Martin I et Stewart D (2001) The differential impact of goal congruency on attitudes, intentions, and the transfer of brand equity. Journal of Marketing Research 38(4): 471-484.

McCroskey J C et Richmond V P (1990) Willingness to communicate: Differing cultural perspectives. Southern Journal of Communication, 56(1): 72-77.

Medin D, Goldstone R et Gentner D (1990) Similarity involving attributes and relations: judgments of similarity and difference are not inverses. Psychological Science 1(1): 64-69.

Medin D, Goldstone R et Gentner D (1993) Respects for similarity. Psychological Review 100 (2): 254-278.

Medin D et Smith E (1984) Concepts and concept formation. Annual Review of Psychology, 35: 113-138.

Mednick S et Mednick M (1962) Remote associates test. Boston: Houghton Mifflin.

Meyers-Levy J, et Tybout A (1989) Schema congruity as a basis for product evaluation. Journal of Consumer Research 16 (1): 39-54.

Mercanti-Guérin M (2010) L'écosystème des sites de vente en ligne : une analyse par le Web Analytics. XII conférence Etienne Thil, La Rochelle, France, Octobre. Meyers-Levy J, Louie T et Curren M (1994) How does the congruity of brand names affect evaluations of brand name extensions? Journal of Applied Psychology 79(1): 46-53.

Monga A et Gürhan-Canli Z (2012) The influence of mating mind-sets on brand extension evaluation. Journal of Marketing Research 49(4): 581-593.

Monga A B et John DR (2010) What makes brands elastic? The influence of brand concept and styles of thinking on brand extension evaluation. Journal of Marketing 74 (3) : 80-92.

Monneret P, (2014) Motivation et analogie, Enjeux de la similarité en sciences du langage. Philologia, 2011(56) : 27-38.

Moon Y (2000) Intimate exchanges: using computers to elicit self-disclosure from consumers. Journal of Consumer Research 26(4): 323-339.

Mori M (1970) The uncanny valley. Energy 7: 33-35.

Murphy G et Medin D (1985) The role of theories in conceptual coherence. Psychological Review 92(3): 289-316.

Nass C, Moon Y, Fogg B, Reeves B et Dryer C (1995) Can computer personalities be human personalities? International Journal of Human-Computers Studies 43( 2): 223-239.

Neidell L (1972) Procedures for obtaining similarities data. Journal of Marketing Research 9(3): 335-337.

Nowak K (2004) The influence of anthropomorphism and agency on social judgment in virtual environments. Journal of Computer Mediated Communication 9(2). 
Ortony A (1979) Beyond literal similarity. Psychological Review 86 (3): 161-180.

Ozanne J, Brucks M et Grewal D (1992) A study of information search behavior during the categorization of new products. Journal of Consumer Research 18(4): 452-463.

Park C, Lawson R et Milberg S (1989) Memory structure of brand names. Advances in Consumer Research 16 : 726-731.

Park C, Milberg S et Lawson R (1991) Evaluation of brand extensions: the role of product feature similarity and brand concept consistency. Journal of Consumer Research 18(2): 185193.

Pavelchak M (1989) Piecemeal and category-based evaluation: an idiographic analysis. Journal of Personality and Social Psychology 56(3): 354-363.

Pham M (1996) Heuristiques et biais décisionnels en marketing. Recherche et Applications en Marketing 11(4) : 53-69.

Poncin I, Pieters R et Ambaye M (2006) Cross-advertisement affectivity: The influence of similarity between commercials and processing modes of consumers on advertising processing. Journal of Business Research 59 (6): 745-754.

Rangaswamy A, Burke R et Oliva T (1993) Brand equity and the extendibility of brand names. International Journal of Research in Marketing 10(1): 61-75.

Ratneshwar S, Barsalou L, Pechmann C et Moore M (2001) Goal-derived categories: the role of personal and situational goals in category representations, Journal of Consumer Psychology 10 (3): 147-157.

Ratneshwar S et Shocker A (1991) Substitution in use and the role of usage context in product category structures. Journal of Marketing Research 28(3): 381-395.

Reed H (1927) The essential laws of learning or association. Psychological Review 34(2): 107115 .

Rips L et Collins A (1993) Categories and resemblance. Journal of Experimental Psychology General 122(4): 468-486.

Rips L, Shoben E et Smith E (1973) Semantic distance and the verification of semantic relations. Journal of Verbal Learning and Verbal Behavior 12(1): 1-20.

Rosch E, Mervis C, Gray W, Johnson D et Boyes-Braem P (1976) Basic objects in natural categories. Cognitive Psychology 8: 382-439.

Saaksjarvi M et Pulkkinen E (2009) The category label and overall similarity in hybrid products: matches/mismatches and categorization asymmetry in consumer preferences. Advances in consumer research 36: 795-796. 
Sar S, Duff B et Anghelcev G (2011) If you feel it now you will think it later: The interactive effects of mood over time on brand extension evaluations. Psychology \& Marketing 28(6): 561583.

Schmitt B et Dubé L (1992) Contextualized representations of brand extensions: Are feature lists or frames the basic components of consumer cognition? Marketing Letters 3(2): 115-126. Schützwohl A (1998) Surprise and schema strength. Journal of Experimental Psychology: Learning, Memory, and Cognition 24(5): 1182-1199.

Schweidel D, Bradlow E et Williams P (2006) A feature-based approach to assessing advertisement similarity. Journal of Marketing Research 43(2): 237-243.

Seltène M (2004) Processus d'évaluation de l'extension de marque par le consommateur: conception et validation d'un modèle de décomposition. Recherche et Applications en Marketin, 19(1): 3-24.

Shanon B (1988) On similarity of features. New Ideas in Psychology 6(3): 307-321.

Shepard R (1962) The analysis of proximities: multidimensional scaling with an unknown distance function. Psychometrika 27(2): 125-140.

Sirgy J (1982) Self-concept in consumer behavior: a critical review. Journal of Consumer Research 9: 287-300.

Smith DC et Park CW (1992) The effects of brand extensions on market share and advertising efficiency. Journal of Marketing Research 29: 296-313.

Stayman D, Alden D et Smith K (1992) Some effects of schematic processing on consumer expectations and disconfirmation judgments. Journal of Consumer Research 19(2): 240-255.

Sujan M (1985) Consumer knowledge: effects on evaluation strategies mediating consumer judgments. Journal of Consumer Research 12(1): 31-46.

Sujan M, Bettman J et Sujan H (1986) Effects of consumer expectations on information processing in selling encounters. Journal of Marketing Research 23(4): 346-353.

Swartz T (1984) Relationship between source expertise and source similarity in an advertising context. Journal of Advertising 13(2): 49-55.

Tauber E (1988) Brand leverage strategy for growth in a cost-control world. Journal of Advertising Research 28(4): 26-30.

Taylor V et Bearden W (2002) The effects of price on brand extension evaluations: the moderating role of extension similarity. Journal of the Academy of Marketing Science 30(2): 131-140.

Thibaut J-P (1997) Similarité et catégorisation. L'année psychologique 97(4) : 701-736. 
Thompson D et Malaviya P (2013) Consumer-generated ads: does awareness of advertising co-creation help or hurt persuasion? Journal of Marketing 77(3): 33-47.

Thompson M et Zanna M (1995) The conflicted individual: personality-based and domainspecific antecedents of ambivalent social attitudes. Journal of Personality 63(2): 259-288.

Tversky A (1977) Features of similarity. Psychological Review 84(4): 327-352.

Tversky A et Gati I (1982) Similarity, separability, and the triangle inequality. Psychological Review 89(2): 123-154.

Van Auken S, Barry T et Bagozzi R (2006) A cross-country construct validation of cognitive age. Journal of the Academy of Marketing Scienc, 34(3): 439-455.

Völckner F et Sattler H (2006) Drivers of brand extension success. Journal of Marketing $70(2): 18-34$.

Watchravesringkan K, Yan R-N et Yurchisin J (2008) Cross-cultural invariance of consumers' price perception measures: Eastern Asian perspective. International Journal of Retail \& Distribution Management 36(10): 759-779.

Wilkes R et Wilcox J (1977) On the validity and reliability of direct similarity judgements: A comment. Journal of Marketing Research 14(2): 261-262.

Wilkie W et Farris P (1975) Comparison advertising: Problems and potential. Journal of Marketing 39(4): 7-15.

Zajonc R (1980) Feelings and thinking: preferences need no inferences. American Psychologist 35(2): 151-175.

Zhang X, Li S, Burke R et Leykin A (2014) An examination of social influence on shopper behavior using video tracking data. Journal of Marketing 78(5): 24-41. 